\title{
Non-commutative Banach Function Spaces
}

\author{
Ben de Pagter
}

\section{Introduction}

In this paper we survey some aspects of the theory of non-commutative Banach function spaces, that is, spaces of measurable operators associated with a semifinite von Neumann algebra. These spaces are also known as non-commutative symmetric spaces. The theory of such spaces emerged as a common generalization of the theory of classical ("commutative") rearrangement invariant Banach function spaces (in the sense of W.A.J. Luxemburg and A.C. Zaanen) and of the theory of symmetrically normed ideals of bounded linear operators in Hilbert space (in the sense of I.C. Gohberg and M.G. Krein). These two cases may be considered as the two extremes of the theory: in the first case the underlying von Neumann algebra is the commutative algebra $L_{\infty}$ on some measure space (with integration as trace); in the second case the underlying von Neumann algebra is $B(\mathcal{H})$, the algebra of all bounded linear operators on a Hilbert space $\mathcal{H}$ (with standard trace). Important special cases of these non-commutative spaces are the non-commutative $L_{p}$-spaces, which correspond in the commutative case with the usual $L_{p}$-spaces on a measure space, and in the setting of symmetrically normed operator ideals they correspond to the Schatten $p$-classes $\mathfrak{S}_{p}$.

In the present paper we take the Banach function spaces as our point of departure. As will become clear, there are many results in the general theory which are direct analogues of the corresponding results in the classical theory. But, we hasten to say that the proofs are quite different in most cases (partly due to the lack of lattice structure in the non-commutative situation). However, there are also many instances where the non-commutative situation essentially differs from the commutative setting (this is in particular illustrated by some of the results in Section 8, concerning the continuity of so-called operator functions).

Definitions and results are stated in detail, but most of the proofs are omitted (with references to the relevant literature). Some proofs have been included, in particular of results which have not yet appeared in print, as well as some relatively short arguments.

In the Section 2 we review some of the basic features of the classical (that is, commutative) Banach function spaces associated with a measure space. We assume that the reader is familiar with the terminology of the theory of Riesz spaces and Banach lattices (as may be found in e.g. [1], [40]). In particular we discuss rearrangement invariant spaces and the so-called Köthe duality. In Section 3 we review some basic facts concerning von Neumann algebras and in Section 4 we discuss in some detail the $\tau$-measurable operators (associated with a semi-finite von Neumann algebra $\mathcal{M}$ equipped with trace $\tau$ ). Particular attention will be given to the properties of the order structure of the (selfadjoint part of the) space $S(\tau)$ of all such $\tau$-measurable operators. The measure 
topology on the space $S(\tau)$ is introduced and its properties are discussed in Section 5. Again we will digress somewhat on the interplay between the order structure and the topology.

An important role in the theory of non-commutative spaces is played by the generalized singular value function (of a $\tau$-measurable operator). In the commutative theory this corresponds to the so-called decreasing rearrangement of a function and, in the setting of compact operators in Hilbert space, to the sequence of singular values of an operator. The properties of the generalized singular value function are discussed in Section 6 .

Non-commutative Banach function spaces are defined in Section 7 and some of their basic properties are reviewed and some aspects of the duality theory are discussed (in particular, trace duality and the Köthe dual). We end the paper with a brief introduction to the study of so-called operator functions in Section 8.

\section{Banach Function Spaces}

Let $(X, \Sigma, \nu)$ be a measure space. We always assume that $(X, \Sigma, \nu)$ is Maharam, that is, it has the finite subset property (i.e., for every $A \in \Sigma$ with $\nu(A)>0$ there exists $B \in \Sigma$ such that $B \subseteq A$ and $0<\nu(B)<\infty$ ) and is localizable (i.e., the measure algebra is a complete Boolean algebra; recall that the measure algebra of $(X, \Sigma, \nu)$ is obtained from $\Sigma$ by identifying sets which are $\nu$-almost equal). Note that any $\sigma$-finite measure space is a Maharam measure space.

The complex Riesz space of all complex valued measurable $\Sigma$-measurable functions on $X$ (with identification of $\nu$-a.e. equal functions) is denoted by $L_{0}(\nu)$. Since we assume $(X, \Sigma, \nu)$ to be Maharam, $L_{0}(\nu)$ is Dedekind complete.

Definition 2.1 A Banach function space on $(X, \Sigma, \nu)$ is an ideal $E \subseteq L_{0}(\nu)$ (that is, $E$ is a linear subspace of $L_{0}(\nu)$ with the additional property that $f \in$ $L_{0}(\nu), g \in E$ and $|f| \leq|g|$ imply $f \in E$ ) equipped with a norm $\|\cdot\|_{E}$ such that $\left(E,\|\cdot\|_{E}\right)$ is a Banach lattice.

Evidently, any Banach function space is Dedekind complete. We may, and shall, always assume that the carrier of $E$ is equal to $X$ (that is, for every $A \in \Sigma$ with $\nu(A)>0$ there exists $B \in \Sigma$ such that $B \subseteq A, \nu(B)>0$ and $\left.\chi_{B} \in E\right)$. Examples of Banach function spaces are the $L_{p}$-spaces $(1 \leq p \leq \infty)$, Orlicz spaces, Lorentz spaces, and Marcinkiewicz spaces. A concise introduction in the theory of Banach function spaces can be found in Chapter 15 of the book [39] (as in most of the literature on Banach function spaces, the underlying measure space is assumed to be $\sigma$-finite; for a treatment in the setting of more general measure spaces, we refer the reader to [17]). In this paper we will be interested mainly in a special class of Banach function spaces, the so-called rearrangement invariant Banach function spaces.

\subsection{Rearrangements}

For $f \in L_{0}(\nu)$ its distribution function $d_{f}:[0, \infty) \rightarrow[0, \infty]$ is defined by

$$
d_{f}(\lambda)=\nu(\{x \in X:|f(x)|>\lambda\}), \quad \lambda \geq 0 .
$$


Note that $d_{f}$ is decreasing and right-continuous. We are interested only in those functions $f \in L_{0}(\nu)$ for which there exists $\lambda_{0} \geq 0$ such that $d_{f}\left(\lambda_{0}\right)<\infty$, which implies that $\lim _{\lambda \rightarrow \infty} d_{f}(\lambda)=0$. We define

$$
S(\nu)=\left\{f \in L_{0}(\nu): \exists \lambda_{0} \geq 0 \text { s.t. } d_{f}\left(\lambda_{0}\right)<\infty\right\} .
$$

If $f \in L_{0}(\nu)$, then $f \in S(\nu)$ if and only if $f$ is bounded except on a set of finite measure. Evidently, $S(\nu)$ is an ideal in $L_{0}(\nu)$.

For $f \in S(\nu)$ the decreasing rearrangement $\mu(f):[0, \infty) \rightarrow[0, \infty]$ of $|f|$ is defined by

$$
\mu(f ; t)=\inf \left\{\lambda \geq 0: d_{f}(\lambda \leq t)\right\}, \quad t \geq 0 .
$$

Observe the following simple properties of the function $\mu(f)$.

Lemma 2.2 If $f \in S(\nu)$, then

(i). $\mu(f ; t)<\infty$ for all $t>0$;

(ii). $\mu(f)$ is decreasing and right-continuous;

(iii). $\mu(f ; 0)<\infty$ if and only if $f \in L_{\infty}(\nu)$ and in this case $\mu(f ; 0)=\|f\|_{\infty}$;

(iv). the functions $f$ and $\mu(f)$ have the same distribution function, that is, $d_{\mu(f)}=d_{f}$ on $[0, \infty)$ (where $d_{\mu(f)}$ is computed with respect to Lebesgue measure on $[0, \infty))$.

For a detailed account of the properties of decreasing rearrangements of functions we refer the reader to the books [3] and [24]. The decreasing rearrangement of $|f|$ is frequently denoted by $f^{*}$. However, in the setting of the present paper we prefer the notation $\mu(f)$, in particular since the $*$ will be used later on to indicate the adjoints of Hilbert space operators.

Furthermore, we note that

$$
\int_{X}|f| d \nu=\int_{0}^{\infty} \mu(f ; t) d t
$$

for all $f \in S(\nu)$. If $f \in S(\nu)$ and if $\varphi:[0, \infty) \rightarrow[0, \infty)$ is continuous and increasing, then $\mu(\varphi \circ|f|)=\varphi \circ \mu(|f|)$, which implies in particular that $\mu\left(|f|^{p}\right)=\mu(f)^{p}, 1 \leq p<\infty$. Consequently,

$$
\int_{X}|f|^{p} d \nu=\int_{0}^{\infty} \mu(f ; t)^{p} d t, \quad 1 \leq p<\infty,
$$

for all $f \in S(\nu)$.

\subsection{Rearrangement invariant Banach Function Spaces}

Let $E$ be a Banach function space on the Maharam measure space $(X, \Sigma, \nu)$.

Definition 2.3 The Banach function space $E \subseteq S(\nu)$ is called rearrangement invariant if $f \in E, g \in S(\nu)$ and $\mu(g)=\mu(f)$ imply that $g \in E$ and $\|g\|_{E}=$ $\|f\|_{E}$. 
Rearrangement invariant spaces are discussed in e.g. [3], [24] or [27] (however, the results in [3] should be handled with some care, as the class of Banach function spaces considered is more restrictive: the so-called Fatou property is included in their definition of a Banach function space!). For rearrangement invariant function spaces on non- $\sigma$-finite measure spaces, see [17].

It follows from (2) and Lemma 2.2, (iii) that $L_{p}$-spaces are rearrangement invariant Banach function spaces. Other examples are Orlicz spaces, Lorentz spaces and Marcinkiewicz spaces.

The following two examples are of particular interest. The space

$$
\left(L_{1} \cap L_{\infty}\right)(\nu)=L_{1}(\nu) \cap L_{\infty}(\nu)
$$

equipped with the norm given by

$$
\|f\|_{L_{1} \cap L_{\infty}}=\max \left(\|f\|_{1},\|f\|_{\infty}\right)
$$

is a rearrangement Banach function space. An alternative expression for the norm is given by

$$
\|f\|_{L_{1} \cap L_{\infty}}=\sup _{t>0} \frac{1}{\min (t, 1)} \int_{0}^{t} \mu(f ; s) d s .
$$

The other example is the space

$$
\left(L_{1}+L_{\infty}\right)(\nu)=L_{1}(\nu)+L_{\infty}(\nu),
$$

where the norm is defined by

$$
\|f\|_{L_{1}+L_{\infty}}=\inf \left\{\|g\|_{1}+\|h\|_{\infty}: f=g+h, g \in L_{1}(\nu), h \in L_{\infty}(\nu)\right\} .
$$

This norm is also given by

$$
\|f\|_{L_{1}+L_{\infty}}=\int_{0}^{1} \mu(f ; s) d s, \quad f \in\left(L_{1}+L_{\infty}\right)(\nu) .
$$

If $E$ is an rearrangement invariant Banach function space on $(0, \infty)$ (with respect to Lebesgue measure), then

$$
\left(L_{1} \cap L_{\infty}\right)(0, \infty) \subseteq E \subseteq\left(L_{1}+L_{\infty}\right)(0, \infty),
$$

with continuous embeddings (see e.g. [24], Theorem II.4.1). Actually, these inclusions hold whenever the measure space $(X, \Sigma, \nu)$ is non-atomic or, is atomic with all atoms having equal measure.

\subsection{Submajorization}

Most of the classical rearrangement invariant Banach function spaces satisfy a stronger condition than just being rearrangement invariant. To discuss this stronger property we introduce the following notion. As before, $(X, \Sigma, \nu)$ is a Maharam measure space. 
Definition 2.4 Given $f, g \in S(\nu)$ we say that $f$ is submajorized by $g$ (in the sense of Hardy, Littlewood and Polya), denoted by

$$
f \prec g,
$$

if

$$
\int_{0}^{t} \mu(f ; s) d s \leq \int_{0}^{t} \mu(g ; s) d s, \quad t \geq 0 .
$$

Definition 2.5 A Banach function space $E \subseteq S(\nu)$ is called symmetric if it satisfies the following three conditions:

(a). E is rearrangement invariant;

(b). $L_{1} \cap L_{\infty}(\nu) \subseteq E \subseteq\left(L_{1}+L_{\infty}\right)(\nu)$ with continuous embeddings;

(c). if $f, g \in E$ and $f \prec g$ then $\|f\|_{E} \leq\|g\|_{E}$.

As we have already observed before, any rearrangement invariant Banach function space on the interval $(0, \infty)$ automatically satisfies condition (b) of the above definition. The following theorem exhibits a large class of symmetric Banach function spaces. Recall that the norm $\|\cdot\|_{E}$ on a Banach function space $E$ is called a Fatou norm if $0 \leq f_{\alpha} \uparrow f \in E$ implies that $\left\|f_{\alpha}\right\|_{E} \uparrow\|f\|_{E}$.

Theorem 2.6 (Luxemburg) ([27])If E is a rearrangement invariant Banach function space on $(0, \infty)$ with a Fatou norm, then $E$ is a symmetric Banach function space.

For simplicity, we have formulated the above result only for the measure space $(0, \infty)$. Actually, this result holds for any measure space which is either non-atomic or is atomic with all atoms having equal measure. However, the result of the theorem is not valid for any measure space, as is illustrated by the following simple example.

Example 2.7 Let $X=\{1,2\}$ and define the measure $\nu$ by $\nu(\{1\})=2$ and $\nu(\{2\})=1$. For $E$ we take $\mathbb{C}^{X}=\mathbb{C}^{2}$, equipped with the norm given by $\left\|\left(f_{1}, f_{2}\right)\right\|_{E}=\left|f_{1}\right|+\left|f_{2}\right|$.

The importance of the class of symmetric Banach function spaces is already indicated by the following result: any symmetric Banach function space on $(0, \infty)$ has offspring on every Maharam measure space. For convenience, we denote a Banach function space $E$ on the interval $(0, \infty)$ explicitly by $E(0, \infty)$. The following theorem has been obtained by W.A.J. Luxemburg ([27]) under the assumption that the norm on $E(0, \infty)$ is Fatou.

Theorem 2.8 Let $E(0, \infty)$ be a symmetric Banach function space on $(0, \infty)$ and let $(X, \Sigma, \nu)$ be a Maharam measure space. If we define

$$
E(\nu)=\{f \in S(\nu): \mu(f) \in E(0, \infty)\}
$$

and

$$
\|f\|_{E(\nu)}=\|\mu(f)\|_{E(0, \infty)}, \quad f \in E(\nu),
$$

then $\left(E(\nu),\|\cdot\|_{E(\nu)}\right)$ is a symmetric Banach function space on $(X, \Sigma, \nu)$. 
Another important property which is stronger than symmetry is presented in the next definition.

Definition 2.9 A Banach function space $E \subseteq S(\nu)$ is called fully symmetric if it follows from $f \in S(\nu), g \in E$ and $f \prec g$ that $f \in E$ and $\|f\|_{E} \leq\|g\|_{E}$.

It is easily verified that any fully symmetric Banach function space is symmetric in the sense of Definition 2.5, but not conversely. As was shown by A.P. Calderón ([6]), the fully symmetric Banach function spaces are precisely the exact $\left(L_{1}, L_{\infty}\right)$-interpolation spaces (cf. also [3], Chapter 5 ). In connection with Theorem 2.6, we mention that any rearrangement invariant Banach function space on $(0, \infty)$ with the Fatou property (that is, $0 \leq f_{\alpha} \uparrow f$ in $L_{0}(0, \infty)$, $f_{\alpha} \in E$ and $\sup _{\alpha}\left\|f_{\alpha}\right\|_{E}<\infty$ imply that $f \in E$ and $\left.\left\|f_{\alpha}\right\|_{E} \uparrow\|f\|_{E}\right)$ is fully symmetric, as was shown by Luxemburg ([27]).

\subsection{Köthe duality}

Next we discuss some aspects of the duality theory for Banach function spaces. Given a Banach function space $E$ on a Maharam measure space $(X, \Sigma, \nu)$, the Köthe dual space $E^{\times}$of $E$ is defined by

$$
E^{\times}=\left\{g \in L_{0}(\nu): \int_{X}|f g| d \nu<\infty \forall f \in E\right\} .
$$

Evidently, $E^{\times}$is an ideal in $L_{0}(\nu)$ and it can be shown that the carrier of $E^{\times}$ is equal to $X$. For $g \in E^{\times}$we define the linear functional $\varphi_{g}: E \rightarrow \mathbb{C}$ by

$$
\varphi_{g}(f)=\int_{X} f g d \nu, \quad f \in E .
$$

The functional $\varphi_{g}$ is bounded, that is, $\varphi_{g} \in E^{*}$ and the map $g \longmapsto \varphi_{g}$ is linear and injective. Hence, we may identify $E^{\times}$with a subspace of $E^{*}$. If we define

$$
\|g\|_{E^{\times}}=\left\|\varphi_{g}\right\|_{E^{*}}=\sup \left\{\left|\int_{X} f g d \nu\right|: f \in E,\|f\|_{E} \leq 1\right\}
$$

for all $g \in E^{\times}$, then $\left(E^{\times},\|\cdot\|_{E^{\times}}\right)$is a Banach function space on $(X, \Sigma, \nu)$.

Denoting by $E_{n}^{*}$ the band in $E^{*}$ consisting of all order continuous (or, normal) functionals on $E$, the following result shows the importance of the Köthe dual space (see e.g. [39], Chapter 15).

Theorem 2.10 If $E$ is a Banach function space, then

$$
E_{n}^{*}=\left\{\varphi_{g}: g \in E^{\times}\right\}
$$

In particular, the norm on $E$ is order continuous if and only if

$$
E^{*}=\left\{\varphi_{g}: g \in E^{\times}\right\} .
$$

If $E$ is a rearrangement invariant Banach function space and if the measure space $(X, \Sigma, \nu)$ is either non-atomic or is atomic with all atoms having equal measure, then it can be shown that $\left(E^{\times},\|\cdot\|_{E^{\times}}\right)$is also a rearrangement invariant (and, actually, fully symmetric) Banach function space (cf. [3], Section 2.4). For general measure spaces the following result may be obtained.

Theorem 2.11 If $E$ is a symmetric Banach function space, then $E^{\times}$is a fully symmetric Banach function space. 


\section{Von Neumann Algebras}

In this section we review some relevant notions related to von Neumann algebras. For the details we refer the reader to any of the books [9], [21], [22] or [34]. Given a complex Hilbert space $(\mathcal{H},\langle\cdot, \cdot\rangle)$, we denote by $B(\mathcal{H})$ the algebra of all bounded linear operators on $\mathcal{H}$ equipped with the operator norm. The identity operator on is denoted by $\mathbf{1}$. For any operator $x \in B(\mathcal{H})$ we denote by $x^{*}$ its adjoint. Recall that an operator $a \in B(\mathcal{H})$ satisfying $a^{*}=a$ is called self-adjoint (or, hermitian); the real subspace of $B(\mathcal{H})$ consisting of all self-adjoint operators is denoted by $B(\mathcal{H})_{h}$. An operator $a \in B(\mathcal{H})_{h}$ is said to be positive if $\langle a \xi, \xi\rangle \geq 0$ for all $\xi \in \mathcal{H}$. The collection of all positive operators on $\mathcal{H}$ is denoted by $B(\mathcal{H})^{+}$, which is a proper closed generating cone in $B(\mathcal{H})_{h}$.

Definition 3.1 $A$ von Neumann algebra $\mathcal{M}$ on $\mathcal{H}$ is a subalgebra of $B(\mathcal{H})$ such that:

(i). $\mathcal{M}$ is $*$-closed (that is, $x \in \mathcal{M}$ implies that $x^{*} \in \mathcal{M}$ ) and $\mathbf{1} \in \mathcal{M}$;

(ii). $\mathcal{M}$ is closed in $B(\mathcal{H})$ for the weak operator topology.

For any non-empty subset $\mathcal{A} \subseteq B(\mathcal{H})$ we denote by $\mathcal{A}^{\prime}$ the commutant of $\mathcal{A}$, that is,

$$
\mathcal{A}^{\prime}=\{y \in B(\mathcal{H}): x y=y x \quad \forall x \in \mathcal{A}\} .
$$

If $\mathcal{A}$ is *-closed, then $\mathcal{A}^{\prime}$ is a von Neumann algebra. We denote $\mathcal{A}^{\prime \prime}=\left(\mathcal{A}^{\prime}\right)^{\prime}$, the double commutant of $\mathcal{A}$. The following fundamental result provides an alternative definition of von Neumann algebras.

Theorem 3.2 (Von Neumann's Double Commutant Theorem) $A$ *-subalgebra $\mathcal{M}$ of $B(\mathcal{H})$ is a von Neumann algebra if and only if $\mathcal{M}=\mathcal{M}^{\prime \prime}$.

Evidently, $\mathcal{M}=B(\mathcal{H})$ is a von Neumann algebra. Suppose that $(X, \Sigma, \nu)$ is a Maharam measure space and consider the Hilbert space $\mathcal{H}=L_{2}(\nu)$. For $f \in L_{\infty}(\nu)$ define the multiplication operator

$$
M_{f}: L_{2}(\nu) \rightarrow L_{2}(\nu), \quad M_{f}(g)=f g, g \in L_{2}(\nu) .
$$

Then $M_{f} \in B\left(L_{2}(\nu)\right)$ and $\left\|M_{f}\right\|=\|f\|_{\infty}$. The mapping $f \longmapsto M_{f}$ is an algebraic isomorphism and isometry from $L_{\infty}(\nu)$ into $B\left(L_{2}(\nu)\right)$. Moreover, $M_{f}^{*}=M_{\bar{f}}$, where $\bar{f}$ is the complex conjugate of $f$.

Proposition 3.3 Defining

$$
\mathcal{M}=\left\{M_{f}: f \in L_{\infty}(\nu)\right\}
$$

$\mathcal{M}$ is a commutative von Neumann algebra on the Hilbert space $\mathcal{H}=L_{2}(\nu)$.

Actually, every commutative von Neumann algebra is of this form (see e.g. [9], Chapter I.7). Frequently, the von Neumann algebra $\mathcal{M}=\left\{M_{f}: f \in L_{\infty}(\nu)\right\}$ is identified with the algebra $L_{\infty}(\nu)$.

Given a von Neumann algebra $\mathcal{M} \subseteq B(\mathcal{H})$ we define $\mathcal{M}_{h}=\mathcal{M} \cap B(\mathcal{H})_{h}$ which is a real linear subspace of $\mathcal{M}$, and $\mathcal{M}^{+}=\mathcal{M} \cap B(\mathcal{H})^{+}$, which is a proper closed and generating cone in $\mathcal{M}_{h}$. We consider $\mathcal{M}_{h}$ as an ordered vector space with $\mathcal{M}^{+}$as its positive cone. 
Definition 3.4 A trace $\tau$ on $\mathcal{M}$ is a map $\tau: \mathcal{M}^{+} \rightarrow[0, \infty]$ which is additive, positive homogeneous and unitarily invariant, that is,

$$
\tau\left(u a u^{*}\right)=\tau(a)
$$

for all $a \in \mathcal{M}^{+}$and all unitary $u \in \mathcal{M}$.

Definition 3.5 A trace $\tau: \mathcal{M}^{+} \rightarrow[0, \infty]$ is called:

(i). faithful if $\tau(a)>0$ whenever $0<a \in \mathcal{M}$;

(ii). semi-finite if for every $a \in \mathcal{M}^{+}$with $\tau(a)>0$ there exists $0 \leq b \leq a$ such that $0<\tau(b)<\infty$;

(iii). normal if $\tau\left(a_{\beta}\right) \uparrow \tau(a)$ whenever $a_{\beta} \uparrow a$ in $\mathcal{M}^{+}$.

A von Neumann algebra equipped with a semi-finite faithful normal trace is called a semi-finite von Neumann algebra.

Example 3.6 (i). Let $\mathcal{H}$ be a Hilbert space and $\mathcal{M}=B(\mathcal{H})$. Given a maximal orthonormal system $\left\{e_{\alpha}\right\}$ in $\mathcal{H}$ we define

$$
\tau(a)=\sum_{\alpha}\left\langle a e_{\alpha}, e_{\alpha}\right\rangle, \quad a \in B(\mathcal{H})^{+} .
$$

The value of $\tau(a)$ does not depend on the particular choice of the maximal orthonormal system in $\mathcal{H}$ and $\tau: B(\mathcal{H})^{+} \rightarrow[0, \infty]$ is a semi-finite faithful normal trace on $B(\mathcal{H})$. This is called the standard trace on $B(\mathcal{H})$.

(ii). Let $\mathcal{H}=L_{2}(\nu)$, where $(X, \Sigma, \nu)$ is a Maharam measure space. On $L_{2}(\nu)$ we consider the von Neumann algebra $\mathcal{M}=L_{\infty}(\nu)$ (see Proposition 3.3). If we define $\tau: L_{\infty}(\nu)^{+} \rightarrow[0, \infty]$ by

$$
\tau(f)=\int_{X} f d \nu, \quad 0 \leq f \in L_{\infty}(\nu),
$$

then $\tau$ is a semi-finite faithful normal trace on $L_{\infty}(\nu)$.

An important object in the study of von Neumann algebras is the collection of all orthogonal projections in $\mathcal{M}$, which is denoted by $P(\mathcal{M})$. It is the analogue in non-commutative integration theory of the underlying $\sigma$-algebra in classical integration theory. The partial ordering in $\mathcal{M}_{h}$ induces a partial order in $P(\mathcal{M})$. If $p, q \in P(\mathcal{M})$, then $p \leq q$ if and only if $\operatorname{Ran}(p) \subseteq \operatorname{Ran}(q)$. For any $p, q \in P(\mathcal{M})$ the infimum $p \wedge q \in P(\mathcal{M})$ and supremum $p \vee q \in P(\mathcal{M})$ exists (and are given by the orthogonal projections onto $\operatorname{Ran}(p) \cap \operatorname{Ran}(q)$ and $\overline{\operatorname{Ran}(p)+\operatorname{Ran}(q)}$, respectively). Actually, $P(\mathcal{M})$ is a complete lattice, that is, for each collection $\left\{p_{\alpha}\right\}$ in $P(\mathcal{M})$, the supremum $\bigvee_{\alpha} p_{\alpha}$ and infimum $\bigwedge_{\alpha} p_{\alpha}$ exist (and are given by the projections onto $\overline{\operatorname{span}}_{\alpha}\left\{\operatorname{Ran}\left(p_{\alpha}\right)\right\}$ and $\bigcap_{\alpha} \operatorname{Ran}\left(p_{\alpha}\right)$, respectively). Every $p \in P(\mathcal{M})$ has a complement, given by $p^{\perp}=\mathbf{1}-p$, which satisfies $p \wedge p^{\perp}=0$ and $p \vee p^{\perp}=\mathbf{1}$. Two projections $p, q \in P(\mathcal{M})$ are called equivalent (with respect to $\mathcal{M}$ ), denoted by $p \sim q$, if there exist a partial isometry $v \in \mathcal{M}$ such that $p=v^{*} v$ and $q=v v^{*}$ (that is, $p$ and $q$ are the initial and final projection of $v$, respectively). If $\tau$ is a trace on $\mathcal{M}$, then $p \sim q$ implies that 
$\tau(p)=\tau(q)$. Furthermore, $p$ is said to be majorized by $q$ (relative to $\mathcal{M}$, denoted by $p \precsim q$, if there exists $r \in P(\mathcal{M})$ such that $r \leq q$ and $p \sim r$. Note that $p \precsim q$ implies that $\tau(p) \leq \tau(q)$. A detailed account of this so-called comparison of projections can be found e.g. in [9], Chapter III.1 or [22], Chapter 6). An important fact is that $p-p \wedge q \sim p \vee q-q$ for all $p, q \in P(\mathcal{M})$, which implies in particular that $p \precsim q^{\perp}$ whenever $p \wedge q=0$.

\section{Measurable operators}

As is clear from the definitions, the space of all measurable functions on a measure space provides the general framework for the theory of Banach function spaces. Analogously, the space of all measurable operators is the setting for theory of non-commutative Banach function spaces and non-commutative integration. These measurable operators are in general unbounded linear operators (think of unbounded measurable functions acting via multiplication on the space $\left.L_{2}(\nu)\right)$. Therefore we first recall some facts about unbounded linear operators in Hilbert space (see e.g. [4] or [21], [22]).

A linear operator in a Hilbert space $\mathcal{H}$ is a linear map $x: \mathcal{D}(x) \rightarrow \mathcal{H}$, where the domain $\mathcal{D}(x)$ is a linear subspace of $\mathcal{H}$. If $\mathcal{D}(x)$ is dense in $\mathcal{H}$, then we say that $x$ is densely defined. The operator $x$ is called closed whenever its graph is a closed subspace of $\mathcal{H} \times \mathcal{H}$. Any closed and densely defined linear operator has a closed and densely defined adjoint $x^{*}: \mathcal{D}\left(x^{*}\right) \rightarrow \mathcal{H}$, which is uniquely determined by the relation $\langle x \xi, \eta\rangle=\left\langle\xi, x^{*} \eta\right\rangle, \xi \in \mathcal{D}(x), \eta \in \mathcal{D}\left(x^{*}\right)$. Note that $x^{* *}=x$.

A closed densely defined linear operator $a: \mathcal{D}(a) \rightarrow \mathcal{H}$ is called self-adjoint if $a^{*}=a$ (meaning that also the domains coincide). If in addition $\langle a \xi, \xi\rangle \geq 0$ for all $\xi \in \mathcal{D}(\mathcal{H})$, then $a$ is said to be positive (which is also denoted by $a \geq 0$ ). For every self-adjoint operator $a$ there exists a unique spectral measure $e^{a}: \mathcal{B}(\mathbb{R}) \rightarrow$ $B(\mathcal{H})$ (that is, $e^{a}$ takes its values in the orthogonal projections and is $\sigma$-additive with respect to the strong operator topology) such that

$$
a=\int_{\mathbb{R}} \lambda d e^{a}(\lambda)
$$

as a spectral integral. Here, $\mathcal{B}(\mathbb{R})$ is the Borel $\sigma$-algebra of $\mathbb{R}$. The spectral measure $e^{a}$ is actually supported on the spectrum $\sigma(a)$ of $a$. In particular, if $a \geq 0$, then $e^{a}$ is supported on $[0, \infty)$. Using the spectral measure of $a$ we may define the Borel functional calculus for $a$ : for any Borel function $f: \sigma(a) \rightarrow \mathbb{C}$ the operator $f(a)$ is defined by

$$
f(a)=\int_{\sigma(a)} f(\lambda) d e^{a}(\lambda),
$$

which is normal operator on $\mathcal{H}$ (recall that the closed and densely defined operator $x$ is called normal whenever $x x^{*}=x^{*} x$, with equality of domains). In particular, if $a \geq 0$, then the (positive) square root of $a$ is given by $a^{1 / 2}=$ $\int_{[0, \infty)} \lambda^{1 / 2} d e^{a}(\lambda)$. It can be shown that $a^{1 / 2}$ is the unique positive operator satisfying $\left(a^{1 / 2}\right)^{2}=a$.

If $x: \mathcal{D}(x) \rightarrow \mathcal{H}$ is a closed densely defined linear operator, then it can be shown that the operator $x^{*} x$ is self-adjoint and actually, positive. The modulus 
$|x|$ of $x$ is defined by

$$
|x|=\left(x^{*} x\right)^{1 / 2},
$$

that is,

$$
|x|=\int_{[0, \infty)} \sqrt{\lambda} d e^{x^{*} x}(\lambda) .
$$

The operator $x$ can be written as

$$
x=v|x|,
$$

where $v$ is a partial isometry. This is called the polar decomposition of $x$.

Now we are ready to introduce the notion of measurable operator (the details may be found in e.g. [36] or [35], Chapter IX). We assume that $(\mathcal{M}, \tau)$ is a semifinite von Neumann algebra on the Hilbert space $\mathcal{H}$, with a fixed faithful normal semi-finite trace $\tau$. A linear operator $x: \mathcal{D}(x) \rightarrow \mathcal{H}$ is called affiliated with $\mathcal{M}$, if $u x=x u$ for all unitary $u \in \mathcal{M}^{\prime}$. This is denoted by $x \eta \mathcal{M}$. Note that the equality $u x=x u$ involves in particular equality of the domains of the operators $u x$ and $x u$, that is, $\mathcal{D}(x)=u^{-1}(\mathcal{D}(x))$. If $x \in B(\mathcal{H})$, then $x$ is affiliated with $\mathcal{M}$ if and only if $x \in \mathcal{M}$ (as follows from Von Neumann's Double Commutant Theorem; see Theorem 3.2). A useful characterization of affiliated operators is presented in the next proposition.

Proposition 4.1 If $x: \mathcal{D}(x) \rightarrow \mathcal{H}$ is a closed and densely defined linear operator with polar decomposition $x=v|x|$, then $x$ is affiliated with $\mathcal{M}$ if and only if:

(i). $e^{|x|}(B) \in \mathcal{M}$ for all $B \in \mathcal{B}(\mathbb{R})$;

(ii). $v \in \mathcal{M}$.

If $\mathcal{M}=B(\mathcal{H})$, then it is clear that every closed and densely defined linear operator $x$ in $\mathcal{H}$ is affiliated with $B(\mathcal{H})$. Hence, the affiliated operators do not have any reasonable algebraic structure in general. To obtain this we further restrict the class of operators to be considered.

Definition 4.2 A closed and densely defined linear operator $x: \mathcal{D}(x) \rightarrow \mathcal{H}$ is called $\tau$-measurable if:

(a). $x \eta \mathcal{M}$;

(b). there exists $\lambda \geq 0$ such that $\tau\left(e^{|x|}(\lambda, \infty)\right)<\infty$.

Condition (b) in the above definition guarantees that the domain of the operator $x$ is "reasonably large" (with respect to the trace $\tau$ ). In fact, if a closed operator $x: \mathcal{D}(x) \rightarrow \mathcal{H}$ is affiliated with $\mathcal{M}$, then $x$ is $\tau$-measurable if and only if its domain $\mathcal{D}(x)$ is $\tau$-dense in $\mathcal{H}$ (that is, there exists a sequence $\left\{p_{n}\right\}_{n=1}^{\infty}$ of orthogonal projections in $\mathcal{M}$ such that $p_{n}(\mathcal{H}) \subseteq \mathcal{D}(x)$ for all $n$, $p_{n} \uparrow \mathbf{1}$ and $\tau\left(\mathbf{1}-p_{n}\right) \downarrow 0$ as $\left.n \rightarrow \infty\right)$.

The collection of all $\tau$-measurable operators is denoted by $S(\tau)$. If $x, y \in$ $S(\tau)$, then the algebraic sum $x+y$ and product $x y$ need not be $\tau$-measurable: these may fail to be closed. However, it can be shown that the operators $x+y$ and $x y$ are closable and that there closures, $x \hat{+} y$ and $x \hat{\cdot y}$ (called the strong sum and strong product, respectively) are $\tau$-measurable. Moreover, if $x \in S(\tau)$, then $x^{*} \in S(\tau)$. All this leads to the following result. 
Theorem 4.3 The set $S(\tau)$ is a complex *-algebra with unit element $\mathbf{1}$, with respect to the operations of strong sum and strong product and the *-operation of taking adjoints. The von Neumann algebra $\mathcal{M}$ is a *-subalgebra of $S(\tau)$.

From now on we denote the strong sum $x \hat{+} y$ and product $\hat{x} \cdot y$ of two elements $x, y \in S(\tau)$ simply by $x+y$ and $x y$, respectively.

Example 4.4 (i). If $\mathcal{M}=B(\mathcal{H})$ with standard trace $\tau$ (see Example $3.6(i)$ ), then $S(\tau)=B(\mathcal{H})$.

(ii). If $\mathcal{H}=L_{2}(\nu), \mathcal{M}=L_{\infty}(\nu)$ and $\tau(f)=\int_{X} f d \nu, 0 \leq f \in L_{\infty}(\nu)$ (see Example 3.6 (ii)), then $S(\tau)=S(\nu)$ (see (1)), where the functions in $S(\nu)$ are identified with (in general unbounded) multiplication operators on $L_{2}(\nu)$.

The real subspace of $S(\tau)$ consisting of all self-adjoint elements is denoted by $S_{h}(\tau)$. Note that $S(\tau)=S_{h}(\tau) \oplus i S_{h}(\tau)$. Indeed, any $x \in S(\tau)$ can be written as $x=\operatorname{Re}(x)+i \operatorname{Im}(x)$, where $\operatorname{Re}(x)=1 / 2\left(x+x^{*}\right)$ and $\operatorname{Im}(x)=1 / 2 i\left(x-x^{*}\right)$. The set of all positive elements in $S_{h}(\tau)$, denoted by $S_{h}(\tau)^{+}$, is a proper cone in $S_{h}(\tau)$. For $a, b \in S_{h}(\tau)$ we define $a \leq b$ whenever $b-a \in S_{h}(\tau)^{+}$. With respect to this ordering, $S_{h}(\tau)$ is a partially ordered vector space. Evidently, this partial ordering is an extension of the ordering in $\mathcal{M}_{h}$. For every $a \in S_{h}(\tau)$ the operators $a^{+}=\int_{\mathbb{R}} \lambda^{+} d e^{a}(\lambda)$ and $a^{-}=\int_{\mathbb{R}} \lambda^{-} d e^{a}(\lambda)$ belong to $S_{h}(\tau)^{+}$and satisfy $a=a^{+}-a^{-}$. Consequently, the positive cone $S_{h}(\tau)^{+}$is generating in $S_{h}(\tau)$. In the next proposition we collect some simple properties of the partial ordering in $S_{h}(\tau)$ (cf. [12]).

Proposition 4.5 (i). If $a, b \in S_{h}(\tau)^{+}$, then $a \leq b$ if and only if $\mathcal{D}\left(b^{1 / 2}\right) \subseteq$ $\mathcal{D}\left(a^{1 / 2}\right)$ and $\left\|a^{1 / 2} \xi\right\|_{\mathcal{H}} \leq\left\|b^{1 / 2} \xi\right\|_{\mathcal{H}}$ for all $\xi \in \mathcal{D}\left(b^{1 / 2}\right)$.

(ii). If $a, b \in S_{h}(\tau)^{+}$, then $a \leq b$ if and only if there exists $x \in \mathcal{M}$ such that $a^{1 / 2}=x b^{1 / 2}$ and $\|x\|_{B(\mathcal{H})} \leq 1$

(iii). If $a \leq b$ in $S_{h}(\tau)$ and $x \in S(\tau)$, then $x^{*} a x \leq x^{*} b x$.

(iv). If $a \in S_{h}(\tau)^{+}$is invertible in $S(\tau)$, then $a^{-1} \geq 0$.

(v). If $0 \leq a \leq b$ in $S_{h}(\tau)$ and $a$ is invertible in $S(\tau)$, then $b$ is invertible in $S(\tau)$ and $0 \leq b^{-1} \leq a^{-1}$.

As (i) of the above proposition shows, on $S_{h}(\tau)^{+}$the partial ordering in $S_{h}(\tau)$ coincides with the usual quadratic form ordering of positive operators (see e.g. [23], Section VI.2.5). Statement (ii) follows almost immediately from (i) and (iii), (vi) are more or less evident. Let us indicate a proof of (v). Since $a^{-1} \geq 0$, it follows that $a^{-1 / 2} \in S_{h}(\tau)^{+}$and so, $\mathbf{1} \leq a^{-1 / 2} b a^{-1 / 2}$. By (i), there exists $x \in \mathcal{M}$ such that $\mathbf{1}=x\left(a^{-1 / 2} b a^{-1 / 2}\right)^{1 / 2}=\left(a^{-1 / 2} b a^{-1 / 2}\right)^{1 / 2} x^{*}$. This shows that $\left(a^{-1 / 2} b a^{-1 / 2}\right)^{1 / 2}$, and hence, $b$ is invertible in $S(\tau)$ with $b^{-1} \geq 0$. Now, it follows from $0 \leq a \leq b$ that $0 \leq b^{-1 / 2} a b^{-1 / 2} \leq \mathbf{1}$. Since $b^{-1 / 2} a b^{-1 / 2}=\left(a^{1 / 2} b^{-1 / 2}\right)^{*}\left(a^{1 / 2} b^{-1 / 2}\right)$, this implies that $\left\|a^{1 / 2} b^{-1 / 2}\right\|_{B(\mathcal{H})} \leq 1$ and so, $\left\|\left(a^{1 / 2} b^{-1 / 2}\right)^{*}\right\|_{B(\mathcal{H})} \leq 1$, which implies that $0 \leq a^{1 / 2} b^{-1} a^{1 / 2} \leq \mathbf{1}$. Using (iii) once again (with $x=a^{-1 / 2}$ ), we may conclude that $0 \leq b^{-1} \leq a^{-1}$. 
Using (i) of Proposition 4.5, one may prove that $S_{h}(\tau)$ is Dedekind complete in the following sense (see Proposition 1.1 in [12]).

Proposition 4.6 If $\left\{a_{\beta}\right\}$ is an increasing net in $S_{h}(\tau)$ and there exists $b \in$ $S_{h}(\tau)$ such that $a_{\beta} \leq b$ for all $\beta$, then $\sup _{\beta} a_{\beta}$ exists in $S_{h}(\tau)$.

Another, related property of the ordering in $S_{h}(\tau)$ is exhibited in the following proposition (see Proposition 1.3 in [12]).

Proposition 4.7 If $\left\{a_{\beta}\right\}$ is an increasing net in $S_{h}(\tau)$ such that $a_{\beta} \uparrow a \in$ $S_{h}(\tau)$, then $x^{*} a_{\beta} x \uparrow x^{*}$ ax for all $x \in S(\tau)$.

Next we discuss the Borel functional calculus (given by (5)) for operators $a \in$ $S_{h}(\tau)$. For this purpose, we denote by $B_{b c}(\sigma(a))$ the $*$-algebra (with respect to complex conjugation) of all complex valued Borel functions on $\sigma(a)$ which are bounded on all compact subsets of $\sigma(a)$. The proof of the first statement of the next proposition may be found in [31], Proposition 3.5; the second statement follows immediately from the properties of the functional calculus.

Proposition 4.8 If $a \in S_{h}(\tau)$, then $f(a) \in S(\tau)$ for all $f \in B_{b c}(\sigma(a))$. Moreover, the map $f \longmapsto f(a)$ is a *-homomorphism from $B_{b c}(\sigma(a))$ into $S(\tau)$ (so, in particular, this map is positive).

\section{$5 \quad$ The measure topology in $S(\tau)$}

The *-algebra $S(\tau)$ of all $\tau$-measurable operators carries an important and useful vector space topology, the so-called $(\tau-)$ measure topology, which is Hausdorff, metrizable and complete (but, not locally convex in general).

As before, $(\mathcal{M}, \tau)$ is a fixed semi-finite von Neumann algebra on a Hilbert space $\mathcal{H}$. For convenience, we denote the set of all orthogonal projections in $\mathcal{M}$ by $P(\mathcal{M})$. Given $0<\varepsilon, \delta \in \mathbb{R}$ we define $V(\varepsilon, \delta)$ to be the set of all $x \in S(\tau)$ for which there exists $p \in P(\mathcal{M})$ such that $\|x p\|_{B(\mathcal{H})} \leq \varepsilon$ and $\tau(\mathbf{1}-p) \leq \delta$. An alternative description of this set is given by

$$
V(\varepsilon, \delta)=\left\{x \in S(\tau): \tau\left(e^{|x|}(\varepsilon, \infty)\right) \leq \delta\right\}
$$

It can be shown that $V(\varepsilon, \delta)$ is balanced and absorbing. Furthermore, for $\varepsilon_{j}, \delta_{j}>0(j=1,2)$ we have $V\left(\varepsilon_{1}, \delta_{1}\right)+V\left(\varepsilon_{2}, \delta_{2}\right) \subseteq V\left(\varepsilon_{1}+\varepsilon_{2}, \delta_{1}+\delta_{2}\right)$ and $V(\varepsilon, \delta) \subseteq V\left(\varepsilon_{1}, \delta_{1}\right) \cap V\left(\varepsilon_{2}, \delta_{2}\right)$, where $\varepsilon=\min \left(\varepsilon_{1}, \varepsilon_{2}\right)$ and $\delta=\min \left(\delta_{1}, \delta_{2}\right)$. These properties imply that the collection $\{V(\varepsilon, \delta)\}_{\varepsilon, \delta>0}$ is a neighbourhood base at 0 for a vector space topology $\mathcal{T}_{m}$ on $S(\tau)$. Since $\bigcap_{\varepsilon \delta>0} V(\varepsilon, \delta)=\{0\}$, this topology is Hausdorff. Moreover, $V\left(\varepsilon_{1}, \delta_{1}\right) V\left(\varepsilon_{2}, \delta_{2}\right) \subseteq V\left(\varepsilon_{1} \varepsilon_{2}, \delta_{1} \delta_{2}\right)$ for all $\varepsilon_{j}, \delta_{j}>0$ and $V(\varepsilon, \delta)^{*}=V(\varepsilon, \delta)$, and so, $S(\tau)$ is also a topological $*$-algebra with respect to $\mathcal{T}_{m}$. The countable subcollection $\{V(1 / n, 1 / n)\}_{n=1}^{\infty}$ is also a base at 0 for $\mathcal{T}_{m}$ and hence, $\mathcal{T}_{m}$ is metrizable. Furthermore, it can be shown that $S(\tau)$ is complete with respect to $\mathcal{T}_{m}$. We collect these results (and some more) in the next theorem (for a proof, see e.g. [36]).

Theorem 5.1 The collection $\{V(\varepsilon, \delta)\}_{\varepsilon, \delta>0}$ is a neighbourhood base at 0 for a metrizable complete Hausdorff vector space topology $\mathcal{T}_{m}$ on $S(\tau)$. With respect to this topology, $S(\tau)$ is a topological *-algebra. Moreover, $\mathcal{M}$ is dense in $S(\tau)$ and the inclusion of $\mathcal{M}$ (with its norm topology) into $S(\tau)$ is continuous. 
The topology $\mathcal{T}_{m}$ is called the measure topology on $S(\tau)$ and convergence with respect to $\mathcal{T}_{m}$ is called convergence in measure (denoted by $x_{n} \stackrel{\mathcal{T}_{m}}{\rightarrow} x$ ). If $\left\{x_{n}\right\}_{n=1}^{\infty}$ is a sequence in $S(\tau)$, then it is immediately clear from (6) that

$$
x_{n} \stackrel{\mathcal{T}_{m}}{\rightarrow} 0 \Longleftrightarrow \lim _{n \rightarrow \infty} \tau\left(e^{\left|x_{n}\right|}(\varepsilon, \infty)\right)=0 \quad \exists \varepsilon>0 .
$$

Furthermore, it is of some interest to note that the neighbourhoods $V(\varepsilon, \delta)$ are actually closed for the measure topology.

Example 5.2 (a). Let $(X, \Sigma, \nu)$ be a Maharam measure space. Let $\mathcal{M}=$ $L_{\infty}(\nu)$, acting via multiplication on $\mathcal{H}=L_{2}(\nu)$, equipped with the trace given by $\tau(f)=\int_{X} f d \nu, f \in L_{\infty}(\nu)^{+}$(see Example 3.6 (ii)). As we have mentioned in Example 4.4, the algebra $S(\tau)$ may be identified with the space $S(\nu)$. Via this identification, the measure topology in $S(\tau)$ corresponds to the usual topology of convergence in measure in $S(\nu)$, a neighbourhood base at 0 of which is given by the sets

$$
\{f \in S(\nu): \nu(x \in X:|f(x)|>\varepsilon) \leq \delta\}, \quad \varepsilon, \delta>0 .
$$

(b). Let $\mathcal{H}$ be any Hilbert space and $\mathcal{M}=B(\mathcal{H})$, equipped with standard trace $\tau$ (see Example 3.6 (ii)). As observed in Example 4.4 (ii), $S(\tau)=B(\mathcal{H})$ in this case. If $e$ is an orthogonal projection with $\tau(\mathbf{1}-e)<1$, then $e=\mathbf{1}$ and so,

$$
V(\varepsilon, \delta)=\left\{x \in B(\mathcal{H}):\|x\|_{B(\mathcal{H})} \leq \varepsilon\right\}
$$

for all $\varepsilon>0$ and $0<\delta<1$. Hence, the measure topology in $S(\tau)=B(\mathcal{H})$ coincides with the operator norm topology in $B(\mathcal{H})$.

Next we discuss the relation between the partial ordering in $S_{h}(\tau)$ and the measure topology. First observe that the map $x \longmapsto \operatorname{Re}(x)$ is (uniformly) continuous (as $\operatorname{Re}(x)=1 / 2\left(x+x^{*}\right)$ ) and so, $S_{h}(\tau)$ is a closed real subspace of $S(\tau)$. Another relevant observation in this respect is that the sets $V(\varepsilon, \delta)$ are absolutely solid: if $x \in V(\varepsilon, \delta)$ and $y \in S(\tau)$ with $|y| \leq|x|$, then $y \in V(\varepsilon, \delta)$. In the next proposition we collect some elementary properties (for the proof of (i) see [12], Proposition 1.4; the other statements follow immediately).

Proposition 5.3 (i). The positive cone $S_{h}(\tau)^{+}$is closed in $S_{h}(\tau)$.

(ii). If $\left\{a_{n}\right\}_{n=1}^{\infty}$ is a sequence in $S_{h}(\tau)$ and $a, b \in S_{h}(\tau)$ are such that $a_{n} \stackrel{\mathcal{T}_{m}}{\rightarrow} a$ and $a_{n} \leq b$ for all $n$, then $a \leq b$.

(iii). If $\left\{a_{n}\right\}_{n=1}^{\infty}$ is an increasing sequence in $S_{h}(\tau)$ and $a_{n} \stackrel{\mathcal{T}_{m}}{\rightarrow} a \in S_{h}(\tau)$, then $a=\sup _{n} a_{n}$ in $S_{h}(\tau)$.

(iv). If $\left\{x_{n}\right\}_{n=1}^{\infty}$ and $\left\{y_{n}\right\}_{n=1}^{\infty}$ are two sequences in $S(\tau)$ such that $y_{n} \stackrel{\mathcal{T}_{m}}{\rightarrow} 0$ and $\left|x_{n}\right| \leq\left|y_{n}\right|$ for all $n$, then $x_{n} \stackrel{\mathcal{T}_{m}}{\rightarrow} 0$.

In some sense, (iii) of the above proposition states that for increasing sequences, measure convergence implies order convergence. What about the converse: does $a_{n} \uparrow a$ in $S_{h}(\tau)$ imply that $a_{n} \stackrel{\mathcal{T}_{m}}{\rightarrow} a$ ? In general not (not even in 
the commutative situation). However, a restricted version is true. To formulate this result, we introduce the subspace $S_{0}(\tau)$ of $S(\tau)$ defined by

$$
S_{0}(\tau)=\left\{x \in S(\tau): \tau\left(e^{|x|}(\lambda, \infty)\right)<\infty \forall \lambda>0\right\} .
$$

In connection with definition (7), recall that for an operator $x \in S(\tau)$ we only know that $\tau\left(e^{|x|}(\lambda, \infty)\right)<\infty$ for some $\lambda>0$ (see Definition 4.2). It can be shown that $S_{0}(\tau)$ is actually a two-sided closed ideal in $S(\tau)$. Moreover, $S_{0}(\tau)$ is absolutely solid in $S(\tau)$, that is, if $y \in S_{0}(\tau), x \in S(\tau)$ and $|x| \leq|y|$, then $x \in S_{0}(\tau)$. The self-adjoint and positive elements in $S_{0}(\tau)$ are denoted by $S_{0, h}(\tau)$ and $S_{0}(\tau)^{+}$respectively. This notation introduced, we can formulate the following "Lebesgue property" of the measure topology.

Proposition 5.4 If $\left\{a_{\beta}\right\}$ is a decreasing net in $S_{h}^{+}(\tau)$ such that $a_{\beta} \downarrow 0$ and if there exists $a \in S_{0}(\tau)^{+}$such that $a_{\beta} \leq a$ for all $\beta$, then $a_{\beta} \stackrel{\mathcal{T}_{m}}{\rightarrow} 0$.

In the sense of the above proposition, one might say that the measure topology on $S_{0, h}(\tau)$ is a "Lebesgue topology" (that is, order convergence implies topological convergence). Next we would like to discuss in some detail some "Fatou type" properties of the measure topology.

Theorem 5.5 Suppose that $\varepsilon, \delta>0, a \in S_{h}(\tau)^{+}$and that $\left\{a_{\beta}\right\}$ is a net in $S_{h}(\tau)^{+}$such that $0 \leq a_{\beta} \uparrow a$ in $S_{h}(\tau)$. If $a_{\beta} \in V(\varepsilon, \delta)$ for all $\beta$, then $a \in$ $V(\varepsilon, \delta)$.

We shall indicate the proof of this result, which is based on the following two technical lemmas. For the notation used we refer to the end of Section 3.

Lemma 5.6 If $a \in S_{h}(\tau)^{+}, 0<\varepsilon \in \mathbb{R}$ and $p \in P(\mathcal{M})$ such that $p \leq e^{a}(\varepsilon, \infty)$, then $p \precsim e^{\text {pap }}(\varepsilon, \infty)$.

Proof. For notational convenience, put $b=$ pap and observe that $b=$ $\left(a^{1 / 2} p\right)^{*}\left(a^{1 / 2} p\right)$ and so, $b^{1 / 2}=\left|a^{1 / 2} p\right|$. Hence, $\mathcal{D}\left(b^{1 / 2}\right)=\mathcal{D}\left(\left|a^{1 / 2} p\right|\right)=$ $\mathcal{D}\left(a^{1 / 2} p\right)$. We first show that $p \wedge e^{b}[0, \varepsilon]=0$. To this end, let $q=p \wedge e^{b}[0, \varepsilon]$ and suppose that $q \neq 0$. Take $\xi \in \mathcal{H}$ such that $q \xi=\xi \neq 0$. This implies that $\xi=e^{b}[0, \varepsilon] \xi=e^{b^{1 / 2}}\left[0, \varepsilon^{1 / 2}\right] \xi$ and so, $\xi \in \mathcal{D}\left(b^{1 / 2}\right)=\mathcal{D}\left(a^{1 / 2} p\right)$. Since $\xi=p \xi$ and the algebraic product of $a^{1 / 2}$ and $p$ is already closed, it follows that $\xi \in$ $\mathcal{D}\left(a^{1 / 2}\right)$. Furthermore, $p \leq e^{a}(\varepsilon, \infty)$ and so, $\xi=e^{a}(\varepsilon, \infty) \xi=e^{a^{1 / 2}}\left(\varepsilon^{1 / 2}, \infty\right) \xi$. Using the properties of spectral measures, it is not difficult to show that this implies that $\left\|a^{1 / 2} \xi\right\|_{\mathcal{H}}>\varepsilon^{1 / 2}\|\xi\|_{\mathcal{H}}$. Hence,

$$
\begin{aligned}
\varepsilon^{1 / 2}\|\xi\|_{\mathcal{H}} & <\left\|a^{1 / 2} \xi\right\|_{\mathcal{H}}=\left\|a^{1 / 2} p \xi\right\|_{\mathcal{H}}=\left\|\left|a^{1 / 2} p\right| \xi\right\|_{\mathcal{H}}=\left\|b^{1 / 2} \xi\right\|_{\mathcal{H}} \\
& =\left\|b^{1 / 2} e^{b^{1 / 2}}\left[0, \varepsilon^{1 / 2}\right] \xi\right\|_{\mathcal{H}} \leq \varepsilon^{1 / 2}\|\xi\|_{\mathcal{H}},
\end{aligned}
$$

which is a contradiction. Therefore, we may conclude that $p \wedge e^{b}[0, \varepsilon]=0$ and this implies that $p \precsim e^{b}[0, \varepsilon]^{\perp}=e^{b}(\varepsilon, \infty)$.

Using this observation we can show that the neighbourhoods $V(\varepsilon, \delta)$ are "locally determined" in the following sense. 
Lemma 5.7 Let $\varepsilon, \delta>0$ be given. If $x \in S(\tau)$, then $x \in V(\varepsilon, \delta)$ if and only if $p|x| p \in V(\varepsilon, \delta)$ for all $p \in P(\mathcal{M})$ with $\tau(p)<\infty$.

Proof. If $x \in V(\varepsilon, \delta)$, then it is easy to see that $p|x| p \in V(\varepsilon, \delta)$ for all $p \in P(\mathcal{M})$ (with $\tau(p)<\infty$ ). For the proof of the converse implication, suppose that $x \notin V(\varepsilon, \delta)$, that is, $\tau\left(e^{|x|}(\varepsilon, \infty)\right)>\delta$. Since the trace is semi-finite, there exists $p \in P(\mathcal{M})$ such that $p \leq e^{|x|}(\varepsilon, \infty)$ and $\delta<\tau(p)<\infty$. By Lemma $5.6, p \precsim e^{p|x| p}(\varepsilon, \infty)$ and so, $\tau\left(e^{p|x| p}(\varepsilon, \infty)\right) \geq \tau(p)>\delta$, which shows that $p|x| p \notin V(\varepsilon, \delta)$.

Now we can provide the proof of Theorem 5.5.

Proof. (of Theorem 5.5) Suppose that $p \in P(\mathcal{M})$ with $\tau(p)<\infty$. It follows from Proposition 4.7 that $0 \leq p a_{\beta} p \uparrow$ pap in $S_{h}(\tau)$. Since $\tau(p)<\infty$, we have $p a p \in S_{0}(\tau)^{+}$, and so, it follows from Proposition 5.4 that $p a_{\beta} p \stackrel{\mathcal{T}_{m}}{\rightarrow} p a p$. Since $p a_{\beta} p \in V(\varepsilon, \delta)$ for all $\beta$ and $V(\varepsilon, \delta)$ is closed for the measure topology, we find that $\operatorname{pap} \in V(\varepsilon, \delta)$. Via Lemma 5.7 we may conclude that $a \in V(\varepsilon, \delta)$.

Recall that a subset $W$ of a topological vector space $(V, \mathcal{T})$ is called bounded if for every neighbourhood $U$ of 0 there exists $0<\lambda \in \mathbb{R}$ such that $W \subseteq \lambda U$. Specializing this notion to the measure topology, we get the following definition.

Definition 5.8 A subset $W$ of $S(\tau)$ is called bounded in measure if for all $\varepsilon, \delta>0$ there exists $\lambda>0$ such that $W \subseteq \lambda V(\varepsilon, \delta)$.

Using that $\lambda V(\varepsilon, \delta)=V(\lambda \varepsilon, \delta)$ for all $\lambda, \varepsilon, \delta>0$ and the definition of the neighbourhoods $V(\varepsilon, \delta)$ we immediately obtain the following characterization of bounded sets in $S(\tau)$.

Proposition 5.9 For a subset $W$ of $S(\tau)$ the following statements are equivalent:

(i). W is bounded in measure;

(ii). for every $\delta>0$ there exists $R>0$ such that $W \subseteq V(R, \delta)$;

(iii). for every $\delta>0$ there exists $R>0$ such that $\tau\left(e^{|x|}(R, \infty)\right) \leq \delta$ for all $x \in W$.

As an example, let us call a set $W \subseteq S(\tau)$ order bounded if there exists $a \in S_{h}(\tau)^{+}$such that $|x| \leq a$ for all $x \in W$. We claim that $W$ is bounded in measure. Indeed, let $\varepsilon, \delta>0$ be given. Since $V(\varepsilon, \delta)$ is absorbing, there exists $\lambda>0$ such that $a \in \lambda V(\varepsilon, \delta)=V(\lambda \varepsilon, \delta)$. Since the set $V(\lambda \varepsilon, \delta)$ is absolutely solid (that is, $y \in V(\lambda \varepsilon, \delta), x \in S(\tau)$ and $|x| \leq|y|$ imply $x \in V(\lambda \varepsilon, \delta)$ ), it is clear that $W \subseteq \lambda V(\varepsilon, \delta)$. Hence, $W$ is bounded in measure. As the next theorem shows, for increasing nets in $S_{h}(\tau)^{+}$, the converse also holds.

Theorem 5.10 If $\left\{a_{\beta}\right\}$ is an increasing net in $S_{h}(\tau)^{+}$which is bounded in measure, then $\sup _{\beta} a_{\beta}$ exists in $S_{h}(\tau)$.

Proof. First we consider a special case. Suppose that $\left\{b_{k}\right\}_{k=1}^{\infty}$ is an increasing sequence of mutually commuting operators (that is, $b_{k} b_{l}=b_{l} b_{k}$ for all $k$ and $l)$ in $S_{h}(\tau)^{+}$which is bounded in measure. We claim that $\sup _{k} b_{k}$ exists in $S_{h}(\tau)$. Indeed, let $\mathfrak{q}_{k}$ be the quadratic form corresponding to the operator $b_{k}$, 
that is, $\mathcal{D}\left(\mathfrak{q}_{k}\right)=\mathcal{D}\left(b_{k}^{1 / 2}\right)$ and $\mathfrak{q}_{k}(\xi)=\left\|b_{k}^{1 / 2} \xi\right\|_{\mathcal{H}}^{2}$ for all $\xi \in \mathcal{D}\left(\mathfrak{q}_{k}\right)$. Defining $\mathfrak{q}: \mathcal{D}(\mathfrak{q}) \rightarrow[0, \infty)$ by

$$
\begin{aligned}
& \mathcal{D}(\mathfrak{q})=\left\{\xi \in \bigcap_{k=1}^{\infty} \mathcal{D}\left(\mathfrak{q}_{k}\right): \sup _{k} \mathfrak{q}_{k}(\xi)<\infty\right\}, \\
& \mathfrak{q}(\xi)=\sup _{k} \mathfrak{q}_{k}(\xi)=\lim _{k \rightarrow \infty} \mathfrak{q}_{k}(\xi), \quad \xi \in \mathcal{D}(\mathfrak{q}),
\end{aligned}
$$

it is easily verified that $\mathfrak{q}$ is a closed quadratic form (in the sense of [23], Section VI.2). The domain $\mathcal{D}(\mathfrak{q})$ is $\tau$-dense (see Section 4 ) in $\mathcal{H}$. To prove this, we have to show that, given $\delta>0$, there exists $p \in P(M)$ such that $p(\mathcal{H}) \subseteq \mathcal{D}(\mathfrak{q})$ and $\tau\left(p^{\perp}\right) \leq \delta$. Since $\left\{b_{k}\right\}_{k=1}^{\infty}$ is bounded in measure, there exists $R>0$ such that $\tau\left(e^{b_{k}}(R, \infty)\right) \leq \delta$ for all $k$. Using that $b_{k} b_{k+1}=b_{k+1} b_{k}$ and $b_{k} \leq b_{k+1}$, it is easily verified that $e^{b_{k}}(R, \infty) \leq e^{b_{k+1}}(R, \infty)$. Therefore, the projection $q=\bigvee_{k=1}^{\infty} e^{b_{k}}(R, \infty)$ satisfies $\tau(q) \leq \delta$. Defining $p=\mathbf{1}-q=\bigwedge_{k=1}^{\infty} e^{b_{k}}[0, R]$ we have $\tau\left(p^{\perp}\right) \leq \delta$ and for $\xi \in p(\mathcal{H})$ we find that

$$
\mathfrak{q}_{k}(\xi)=\left\|b_{k}^{1 / 2} \xi\right\|_{\mathcal{H}}^{2}=\left\|b_{k}^{1 / 2} e^{b_{k}}[0, R] \xi\right\|_{\mathcal{H}}^{2}=\left\|b_{k}^{1 / 2} e^{b_{k}^{1 / 2}}\left[0, R^{1 / 2}\right] \xi\right\|_{\mathcal{H}}^{2} \leq R\|\xi\|_{\mathcal{H}}^{2}
$$

and so, $\sup _{k} \mathfrak{q}_{k}(\xi) \leq R\|\xi\|_{\mathcal{H}}^{2}<\infty$. Hence, $p(\mathcal{H}) \subseteq \mathcal{D}(\mathfrak{q})$, which shows that $\mathcal{D}(\mathfrak{q})$ is $\tau$-dense (and so, norm dense in $\mathcal{H}$ ). Therefore, there exists a unique positive self-adjoint operator $a$ in $\mathcal{H}$ such that $\mathcal{D}\left(a^{1 / 2}\right)=\mathcal{D}(\mathfrak{q})$ and $\left\|a^{1 / 2} \xi\right\|_{\mathcal{H}}^{2}=$ $\mathfrak{q}(\xi)$. Now it is readily verified that $a \in S_{h}(\tau)^{+}$and that $b_{k} \uparrow a$ in $S_{h}(\tau)$.

Now we turn to the general case, where $\left\{a_{\beta}\right\}$ is an increasing net in $S_{h}(\tau)^{+}$ which is bounded in measure. For $k=1,2, \ldots$ we define

$$
Y_{k}\left(a_{\beta}\right)=k a_{\beta}\left(a_{\beta}+k \mathbf{1}\right)^{-1} .
$$

The sequence $\left\{Y_{k}\left(a_{\beta}\right)\right\}_{k=1}^{\infty}$ is called the Yosida approximation of the operator $a_{\beta}$. Note that

$$
\begin{aligned}
Y_{k}\left(a_{\beta}\right) & =k\left(\mathbf{1}-k\left(a_{\beta}+k \mathbf{1}\right)^{-1}\right) \\
& =a_{\beta}-a_{\beta}^{2}\left(a_{\beta}+k \mathbf{1}\right)^{-1} .
\end{aligned}
$$

It is not difficult to show that: (i) $Y_{k}\left(a_{\beta}\right) \in \mathcal{M}$ and $0 \leq Y_{k}\left(a_{\beta}\right) \leq k \mathbf{1}$ for all $k$; (ii) $0 \leq Y_{k}\left(a_{\beta}\right) \leq Y_{k+1}\left(a_{\beta}\right)$ for all $k$; (iii) $Y_{k}\left(a_{\beta}\right) \stackrel{\mathcal{T}_{m}}{\rightarrow} a_{\beta}$ as $k \rightarrow \infty$; (iv) $Y_{k}\left(a_{\beta}\right) \uparrow a_{\beta}$ in $S_{h}(\tau)$; (v) for fixed $k$ we have $Y_{k}\left(a_{\beta}\right) \uparrow_{\beta}$ in $\mathcal{M}$.

Since $0 \leq Y_{k}\left(a_{\beta}\right) \uparrow \leq k \mathbf{1}$ in $\mathcal{M}$, there exists $b_{k} \in \mathcal{M}$ such that $Y_{k}\left(a_{\beta}\right) \uparrow_{\beta}$ $b_{k}$ and $Y_{k}\left(a_{\beta}\right) \rightarrow_{\beta} b_{k}$ with respect to the strong operator topology (that is, $Y_{k}\left(a_{\beta}\right) \xi \rightarrow b_{k} \xi$ for all $\left.\xi \in H\right)$. It is clear that $b_{k} \leq b_{k+1}$ for all $k$. We claim that $b_{k} b_{l}=b_{l} b_{k}$ for all $k, l \geq 1$. Indeed, the nets $\left\{Y_{k}\left(a_{\beta}\right)\right\}_{\beta}$ and $\left\{Y_{l}\left(a_{\beta}\right)\right\}_{\beta}$ are uniformly bounded (by $k$ and $l$, respectively) and converge strongly to $b_{k}$ and $b_{l}$, respectively. This implies that $Y_{k}\left(a_{\beta}\right) Y_{l}\left(a_{\beta}\right) \rightarrow_{\beta} b_{k} b_{l}$ and $Y_{l}\left(a_{\beta}\right) Y_{k}\left(a_{\beta}\right) \rightarrow_{\beta}$ $b_{l} b_{k}$ strongly. Since $Y_{k}\left(a_{\beta}\right) Y_{l}\left(a_{\beta}\right)=Y_{l}\left(a_{\beta}\right) Y_{k}\left(a_{\beta}\right)$ for all $\beta$, we may conclude that $b_{k} b_{l}=b_{l} b_{k}$. Next we show that $\left\{b_{k}\right\}_{k=1}^{\infty}$ is bounded in measure. Let $\delta>0$ be given. Since $\left\{a_{\beta}\right\}$ is bounded in measure, there exists $R>0$ such that $a_{\beta} \in V(R, \delta)$ for all $\beta$. Using that $0 \leq Y_{k}\left(a_{\beta}\right) \leq a_{\beta}$, this implies that $Y_{k}\left(a_{\beta}\right) \in V(R, \delta)$ for all $k \geq 1$ and all $\beta$. Since $Y_{k}\left(a_{\beta}\right) \uparrow_{\beta} b_{k}$ in $S_{h}(\tau)$, it 
follows from Theorem 5.5 that $b_{k} \in V(R, \delta)$ for all $k \geq 1$. Hence, $\left\{b_{k}\right\}_{k=1}^{\infty}$ is bounded in measure.

From the first part of the proof it now follows that there exists $a \in S_{h}(\tau)^{+}$ such that $b_{k} \uparrow a$ in $S_{h}(\tau)$. It is easily verified that also $a_{\beta} \uparrow a$ in $S_{h}(\tau)$, which completes the proof of the theorem.

We end this section mentioning some results concerning the continuity of the functional calculus. It follows from Proposition 4.8 that, for any $a \in S_{h}(\tau)$, the map $f \longmapsto f(a)$ is a $*$-homomorphism from $B_{b c}(\mathbb{R})$ into $S(\tau)$ (here $B_{b c}(\mathbb{R}$ ) denotes the $*$-algebra of all complex valued Borel functions which are bounded on compact subsets of $\mathbb{R}$ ). The following result is relatively easy to prove.

Theorem 5.11 If $f \in B_{b c}(\mathbb{R})$ and $\left\{f_{n}\right\}_{n=1}^{\infty}$ is a sequence in $B_{b c}(\mathbb{R})$ such that $f_{n} \rightarrow f$ uniformly on compact subsets of $\mathbb{R}$, then $f_{n}(a) \stackrel{\mathcal{T}_{m}}{\rightarrow} f(a)$ for all $a \in$ $S_{h}(\tau)$.

The next theorem is less trivial. It is actually a special case of a more general result due to O.Ye. Tikhonov ([37]).

Theorem 5.12 If $f \in C(\mathbb{R})$ and $a_{n} \stackrel{\mathcal{T}_{m}}{\rightarrow} a$ in $S_{h}(\tau)$, then $f\left(a_{n}\right) \stackrel{\mathcal{T}_{m}}{\rightarrow} f(a)$.

Note that this theorem implies in particular that the absolute value map $x \longmapsto|x|$ is continuous on $S(\tau)$ with respect to the measure topology. Indeed, if $x_{n} \stackrel{\mathcal{T}_{m}}{\rightarrow} x$ in $S(\tau)$, then $x_{n}^{*} x_{n} \stackrel{\mathcal{T}_{m}}{\rightarrow} x^{*} x$ and now apply the above theorem with $f(\lambda)=\sqrt{|\lambda|}$.

\section{Generalized singular value functions}

In the setting of $\tau$-measurable operators, the generalized singular value functions are the analogue (and actually, generalization) of the decreasing rearrangements of functions in the classical setting. As before, we assume that $(\mathcal{M}, \tau)$ is a semifinite von Neumann algebra on a Hilbert space $\mathcal{H}$. For $x \in S(\tau)$ the distribution function $d_{x}:[0, \infty) \rightarrow[0, \infty]$ is defined by

$$
d_{x}(\lambda)=\tau\left(e^{|x|}(\lambda, \infty)\right), \quad \lambda \geq 0 .
$$

Note that it follows from the definition of $\tau$-measurability that for each $x \in S(\tau)$ there exists $\lambda_{0} \geq 0$ such that $d_{x}(\lambda)<\infty$ for all $\lambda>\lambda_{0}$. Furthermore, the function $d_{x}$ is decreasing and right-continuous and $\lim _{\lambda \rightarrow \infty} d_{x}(\lambda)=0$.

For $x \in S(\tau)$ the generalized singular value function $\mu(x):[0, \infty) \rightarrow[0, \infty]$ is defined by

$$
\mu(x ; t)=\inf \left\{\lambda \geq 0: d_{x}(\lambda) \leq t\right\}, \quad t \geq 0 .
$$

Since $\lim _{\lambda \rightarrow \infty} d_{x}(\lambda)=0$, it is clear that $\mu(x ; t)<\infty$ for all $t>0$ (and note that $\mu(x ; 0)<\infty$ if and only if $x \in \mathcal{M}$, in which case $\left.\mu(x ; 0)=\|x\|_{B(\mathcal{H})}\right)$. The function $\mu(x)$ is decreasing and right-continuous. The notion of generalized singular value function for operators $x \in \mathcal{M}$ goes back to $\mathrm{A}$. Grothendieck ([19]). A useful alternative description of the function $\mu(x)$ is the following. 
Theorem 6.1 (see [15]) If $x \in S(\tau)$, then

$$
\mu(x ; t)=\inf \left\{\|x p\|_{B(\mathcal{H})}: p \in P(\mathcal{M}), p(\mathcal{H}) \subseteq \mathcal{D}(x), \tau(\mathbf{1}-p) \leq t\right\}
$$

for all $t \geq 0$.

Let us consider two simple examples.

Example 6.2 (i). Let $\mathcal{H}=L_{2}(\nu)$, where $(X, \Sigma, \nu)$ is a Maharam measure space, and $\mathcal{M}=L_{\infty}(\nu)$, equipped with the trace $\tau$ given by $\tau(f)=\int_{X} f d \nu$ for $0 \leq f \in L_{\infty}(\nu)$ (see Example 3.6 (ii)). For any $f \in S(\tau)=S(\nu)$ the generalized singular value function coincides with the decreasing rearrangement as defined in Section 2.1.

(ii). Let $\mathcal{H}$ be any Hilbert space and $\mathcal{M}=B(\mathcal{H})$ equipped with the standard trace $\tau$. If $x \in B(\mathcal{H})$ is a compact operator, then $|x|=\left(x^{*} x\right)^{1 / 2}$ is compact and self-adjoint. The eigenvalues of $|x|$ are called the singular values of $x$, denoted by $\left\{\mu_{n}(x)\right\}_{n=0}^{\infty}$. Here the numbers $\mu_{n}(x)$ are arranged in decreasing order and repeated according to multiplicity, so

$$
\|x\|_{B(\mathcal{H})}=\mu_{0}(x) \geq \mu_{1}(x) \geq \mu_{2}(x) \geq \cdots \downarrow 0 .
$$

It follows from the min-max formulas for the eigenvalues of self-adjoint compact operators (see e.g. [32], Section 95) in combination with Theorem 6.1 that the generalized singular value function of $x$ is given by $\mu(x ; t)=$ $\mu_{n}(x)$ whenever $n \leq t<n+1$ and $n=0,1, \ldots$ This example explains why in the general setting the function $\mu(x)$ is called the generalized singular value function.

There is a close connection between the measure topology and generalized singular value functions. Recall that the neighbourhood base $\{V(\varepsilon, \delta)\}_{\varepsilon, \delta>0}$ at zero for the measure topology is given by

$$
V(\varepsilon, \delta)=\left\{x \in S(\tau): \tau\left(e^{|x|}(\varepsilon, \infty)\right) \leq \delta\right\}
$$

that is,

$$
V(\varepsilon, \delta)=\left\{x \in S(\tau): d_{x}(\varepsilon) \leq \delta\right\}
$$

which implies that

$$
\mu(x ; t)=\inf \{\varepsilon>0: x \in V(\varepsilon, t)\}
$$

for all $t>0$. Conversely, for all $\varepsilon, \delta>0$ we have

$$
V(\varepsilon, \delta)=\{x \in S(\tau): \mu(x ; \delta) \leq \varepsilon\} .
$$

Indeed, if $x \in V(\varepsilon, \delta)$, then it is clear from (8) that $\mu(x ; \delta) \leq \varepsilon$. Conversely, if $\mu(x ; \delta) \leq \varepsilon$, then it follows from the definition of $\mu(x ; \delta)$ and the right-continuity of $d_{x}$ that $d_{x}(\varepsilon) \leq \delta$ and so, $x \in V(\varepsilon, \delta)$. Note that this implies in particular that $x \in V\left(\mu^{\prime}, t\right)$ for all $x \in S(\tau), t>0$ and $\mu^{\prime}>\mu(x ; t)$ (and $x \in V(\mu(x ; t), t)$ whenever $\mu(x ; t)>0)$. These simple observations provide a way to transfer properties of the measure topology to properties of the generalized singular value 
function, and visa versa. For example, in the remarks preceding Proposition 5.3 it has been observed that the sets $V(\varepsilon, \delta)$ are absolutely solid, that is, $x \in V(\varepsilon, \delta), y \in S(\tau)$ and $|y| \leq|x|$, imply that $y \in V(\varepsilon, \delta)$. Using (8), we see that, if $x, y \in S(\tau)$ and $|y| \leq|x|$, then $\mu(y) \leq \mu(x)$. As another example, the property $V(\varepsilon, \delta)^{*}=V(\varepsilon, \delta)$ immediately implies that $\mu\left(x^{*}\right)=\mu(x)$ for all $x \in S(\tau)$. It is not difficult to show that $y V(\varepsilon, \delta) z \subseteq V(\|y\|\|z\| \varepsilon, \delta)$ for all $y, z \in \mathcal{M}$. Consequently, $\mu(y x z) \leq\|y\|\|z\| \mu(x)$ for all $x \in S(\tau)$ and $y, z \in \mathcal{M}$. We present some other examples. In the next proposition we denote $\lim _{s \uparrow t} \mu(x ; s)=\mu(x ; t-0)$ for $t>0$.

Proposition 6.3 (cf. [15], Lemma 3.4) If $x \in S(\tau)$ and $\left\{x_{n}\right\}_{n=1}^{\infty}$ is a sequence in $S(\tau)$ such that $x_{n} \stackrel{\mathcal{T}_{m}}{\rightarrow} x$, then

$$
\mu(x ; t) \leq \liminf _{n \rightarrow \infty} \mu\left(x_{n} ; t\right) \leq \limsup _{n \rightarrow \infty} \mu\left(x_{n} ; t\right) \leq \mu(x ; t-0)
$$

for all $t>0$. In particular, $\mu(x ; t)=\lim _{n \rightarrow \infty} \mu\left(x_{n} ; t\right)$ for any $t>0$ where $\mu(x ; t)$ is continuous (and hence, $\mu\left(x_{n}\right) \rightarrow \mu(x)$ a.e. on $[0, \infty)$ ).

Proof. Since $x_{n} \stackrel{\mathcal{T}_{m}}{\rightarrow} x$, there exist $\varepsilon_{n}>0$ and $\delta_{n}>0$ such that $\varepsilon_{n} \downarrow 0, \delta_{n} \downarrow 0$ and $x-x_{n} \in V\left(\varepsilon_{n}, \delta_{n}\right)$ for all $n$. From the above observations it follows that

$$
\begin{aligned}
x & =x_{n}+\left(x-x_{n}\right) \in V\left(\mu\left(x_{n}, t\right)+\varepsilon_{n}, t\right)+V\left(\varepsilon_{n}, \delta_{n}\right) \\
& \subseteq V\left(\mu\left(x_{n}, t\right)+2 \varepsilon_{n}, t+\delta_{n}\right)
\end{aligned}
$$

and so, $\mu\left(x ; t+\delta_{n}\right) \leq \mu\left(x_{n}, t\right)+2 \varepsilon_{n}$. Since $\mu(x)$ is right-continuous, this implies that $\mu(x ; t) \leq \liminf _{n \rightarrow \infty} \mu\left(x_{n} ; t\right)$. Take $0<s<t$ and let $N \in \mathbb{N}$ be such that $s+\delta_{n} \leq t$ for all $n \geq N$. We find that

$$
\begin{aligned}
x_{n} & =x+\left(x_{n}-x\right) \in V\left(\mu(x ; s)+\varepsilon_{n}, s\right)+V\left(\varepsilon_{n}, \delta_{n}\right) \\
& \subseteq V\left(\mu(x ; s)+2 \varepsilon_{n}, s+\delta_{n}\right) \subseteq V\left(\mu(x ; s)+2 \varepsilon_{n}, t\right)
\end{aligned}
$$

and hence, $\mu\left(x_{n} ; t\right) \leq \mu(x ; s)+2 \varepsilon_{n}$ for all $n \geq N$. This implies that

$$
\limsup _{n \rightarrow \infty} \mu\left(x_{n} ; t\right) \leq \mu(x ; s) .
$$

Letting $s \uparrow t$, we get $\lim \sup _{n \rightarrow \infty} \mu\left(x_{n} ; t\right) \leq \mu(x ; t-0)$.

Corollary 6.4 (see e.g. [12], Lemma 3.5) If $\left\{a_{\beta}\right\}$ is a net in $S_{h}(\tau)^{+}$such that $a_{\beta} \downarrow 0$ in $S_{h}(\tau)$ and there exists $a \in S_{0}(\tau)^{+}$such that $0 \leq a_{\beta} \leq a$ for all $\beta$, then $\mu\left(a_{\beta} ; t\right) \downarrow 0$ for all $t>0$.

Proof. From Proposition 5.4 we know that $a_{\beta} \stackrel{\mathcal{T}_{m}}{\rightarrow} 0$. Since the measure topology is metrizable, there exists a decreasing subsequence $\left\{a_{\beta_{n}}\right\}_{n=1}^{\infty}$ such that $a_{\beta_{n}} \stackrel{\mathcal{T}_{m}}{\rightarrow} 0$. It follows from Proposition 6.3 that $\mu\left(a_{\beta_{n}} ; t\right) \downarrow 0$ as $n \rightarrow \infty$ for all $t>0$, which implies that $\mu\left(a_{\beta} ; t\right) \downarrow 0$ for all $t>0$.

In connection with the above result we mention that the elements in $S_{0}(\tau)$ may be characterized in terms of the generalized singular value function by

$$
S_{0}(\tau)=\{x \in S(\tau): \mu(x ; t) \rightarrow 0 \text { as } t \rightarrow \infty\},
$$

as follows easily from the definition (see (7)). 
Proposition 6.5 (see e.g. [12], Proposition 1.7) If $0 \leq a_{\beta} \uparrow a$ in $S_{h}(\tau)$, then $\mu\left(a_{\beta} ; t\right) \uparrow \mu(a ; t)$ for all $t \geq 0$.

Proof. First we consider the case that $t>0$. Since $\mu\left(a_{\beta} ; t\right) \leq \mu(a ; t)$ for all $\beta$, it is clear that $\alpha=\sup _{\beta} \mu\left(a_{\beta} ; t\right) \leq \mu(a ; t)$. Suppose that $\alpha<\mu(a ; t)$ and take $\alpha_{1} \in \mathbb{R}$ such that $\alpha<\alpha_{1}<\mu(a ; t)$. By $(9), \mu\left(a_{\beta} ; t\right) \leq \alpha_{1}$ implies that $a_{\beta} \in V\left(\alpha_{1}, t\right)$ for all $\beta$. Hence, it follows from Theorem 5.5 that $a \in V\left(\alpha_{1}, t\right)$. Using (9) once again, we find that $\mu(a ; t) \leq \alpha_{1}$, which is a contradiction.

Using that $\mu(a ; 0)=\sup _{t>0} \mu(a ; t)$ and $\mu\left(a_{\beta} ; 0\right)=\sup _{t>0} \mu\left(a_{\beta} ; t\right)$, the case $t=0$ is now an immediate consequence of the above.

Using the generalized singular value function we may also introduce the notion of submajorization (cf. Definition 2.4) for elements of $S(\tau)$. If $x, y \in$ $S(\tau)$, then we say that $x$ is submajorized by $y$, denoted by $x \prec y$, whenever $\mu(x) \prec \mu(y)$, that is,

$$
\int_{0}^{t} \mu(x ; s) d s \leq \int_{0}^{t} \mu(y ; s) d s, \quad t \geq 0 .
$$

There are many useful submajorization inequalities involving the generalized singular value functions of element of $S(\tau)$, analogous to the classical inequalities for functions. We will not even try to list them all here but, we mention two of them for later reference.

Theorem 6.6 If $x, y \in S(\tau)$, then:

(i). $\mu(x+y) \prec \mu(x)+\mu(y)$;

(ii). $\mu(x)-\mu(y) \prec \mu(x-y)$.

Inequality (i) for the case of functions is classical and may probably be traced back to Hardy. Littlewood and Polya. For singular values of compact operators in Hilbert space, (i) was obtained by K. Fan ([16]). The general form for $\tau$-measurable operators is due to Th. Fack and H. Kosaki ([15], Theorem 4.4). For the case of functions, inequality (ii) goes back to G.G. Lorentz and T. Shimogaki ([25]) and for singular values of compact operators in Hilbert space this inequality was obtained by A.S. Markus ([28]). The general case of (ii) was proved in [10].

\section{Non-commutative Banach function spaces}

As before, we assume that $(\mathcal{M}, \tau)$ is a semi-finite von Neumann algebra on a Hilbert space $\mathcal{H}$. Let $E=E(0, \infty)$ be a symmetric Banach function space (see Definition 2.5) on $(0, \infty)$ (with respect to Lebesgue measure). With these ingredients we introduce

$$
\begin{aligned}
E(\tau) & =\{x \in S(\tau): \mu(x) \in E(0, \infty)\} \\
\|x\|_{E(\tau)} & =\|\mu(x)\|_{E(0, \infty)}, x \in E(\tau) .
\end{aligned}
$$

The following result has been obtained in [10], [11]. We shall indicate the main steps of its proof. 
Theorem 7.1 With the above definitions we have:

(i). $E(\tau)$ is a linear subspace of $S(\tau)$ and $\|\cdot\|_{E(\tau)}$ is a norm on $E(\tau)$;

(ii). the embedding of $\left(E(\tau),\|\cdot\|_{E(\tau)}\right)$ into $\left(S(\tau), \mathcal{T}_{m}\right)$ is continuous;

(iii). $E(\tau)$ is complete with respect to $\|\cdot\|_{E(\tau)}$.

Proof. (i). If $x, y \in E(\tau)$, then it follows from Theorem 6.6 (i), that $\mu(x+y) \prec \mu(x)+\mu(y)$, which implies that $x+y \in E(\tau)$ and

$$
\begin{aligned}
\|x+y\|_{E(\tau)} & =\|\mu(x+y)\|_{E(0, \infty)} \leq\|\mu(x)+\mu(y)\|_{E(0, \infty)} \\
& \leq\|\mu(x)\|_{E(0, \infty)}+\|\mu(y)\|_{E(0, \infty)}=\|x\|_{E(\tau)}+\|y\|_{E(\tau)} .
\end{aligned}
$$

Now it is clear that $\|\cdot\|_{E(\tau)}$ is a norm on $E(\tau)$.

(ii). It is sufficient to show that the closed unit ball $B_{E(\tau)}$ of $E(\tau)$ is bounded in measure, that is, for every $\delta>0$ there exists $R>0$ such that $\mu(x ; \delta) \leq R$ whenever $x \in B_{E(\tau)}$ (see Proposition 5.9 and (9)). Given $\delta>0$ and $x \in B_{E(\tau)}$, it follows from the inequality $0 \leq \mu(x ; \delta) \chi_{[0, \delta]} \leq \mu(x)$ (as $\mu(x)$ is decreasing on $[0, \infty))$ that $\mu(x ; \delta) \leq\left\|\chi_{[0, \delta]}\right\|_{E(0, \infty)}^{-1}$. Hence, we may take $R=\left\|\chi_{[0, \delta]}\right\|_{E(0, \infty)}^{-1}$.

(iii). To show that $E(\tau)$ is complete with respect to $\|\cdot\|_{E(\tau)}$, suppose that $\left\{x_{n}\right\}_{n=1}^{\infty}$ is a Cauchy sequence in $E(\tau)$. It follows from (ii) that $\left\{x_{n}\right\}_{n=1}^{\infty}$ is Cauchy for the measure topology and so, there exists $x \in S(\tau)$ such that $x_{n} \stackrel{\mathcal{T}_{m}}{\rightarrow} x$ (see Theorem 5.1). Moreover, it follows from Theorem 6.6 (ii), that

$$
\mu\left(x_{m}\right)-\mu\left(x_{n}\right) \prec \mu\left(x_{m}-x_{n}\right)
$$

and so, $\left\|\mu\left(x_{m}\right)-\mu\left(x_{n}\right)\right\|_{E(0, \infty)} \leq\left\|\mu\left(x_{m}-x_{n}\right)\right\|_{E(0, \infty)}=\left\|x_{m}-x_{n}\right\|_{E(\tau)}$ for all $m$ and $n$. Hence, $\left\{\mu\left(x_{n}\right)\right\}_{n=1}^{\infty}$ is a Cauchy sequence in $E(0, \infty)$. Hence, there exists $f \in E(0, \infty)$ such that $\left\|\mu\left(x_{n}\right)-f\right\|_{E(0, \infty)} \rightarrow 0$. Furthermore, it follows from Proposition 6.3 that $x_{n} \stackrel{\mathcal{T}_{m}}{\rightarrow} x$ implies that $\mu\left(x_{n}\right) \rightarrow \mu(x)$ a.e. on $(0, \infty)$ and so, $\mu(x)=f \in E(0, \infty)$, that is, $x \in E(\tau)$. Applying the same argument to the Cauchy sequence $\left\{x-x_{n}\right\}_{n=1}^{\infty}$ (which converges to 0 in measure), we find that $\left\|\mu\left(x-x_{n}\right)\right\|_{E(0, \infty)} \rightarrow 0$, that is, $\left\|x-x_{n}\right\|_{E(\tau)} \rightarrow 0$. The proof is complete.

The space $E(\tau)$ is called the non-commutative Banach function space corresponding to $E(0, \infty)$ and associated with $(\mathcal{M}, \tau)$. From the definition it is clear that $x \in E(\tau)$ if and only if $|x| \in E(\tau)$, if and only if $x^{*} \in E(\tau)$ and that $\|x\|_{E(\tau)}=\||x|\|_{E(\tau)}=\left\|x^{*}\right\|_{E(\tau)}$. Furthermore we note that $E(\tau)$ is symmetric, that is, if $x \in S(\tau), y \in E(\tau)$ and $x \prec y$, then $x \in E(\tau)$ and $\|x\|_{E(\tau)} \leq\|y\|_{E(\tau)}$ (and so, in particular, $E(\tau)$ is an absolutely solid subspace of $S(\tau)$ ).

The real linear subspace of all self-adjoint elements in $E(\tau)$ is denoted by $E_{h}(\tau)$. The collection of all positive elements in $E_{h}(\tau)$ is denoted by $E_{h}(\tau)^{+}$, that is, $E_{h}(\tau)^{+}=E(\tau) \cap S_{h}(\tau)^{+}$, which is a proper cone in $E_{h}(\tau)$. Hence, $E_{h}(\tau)$ has the structure of a partially ordered vector space with $E_{h}(\tau)^{+}$as its positive cone. Since the embedding of $\left(E(\tau),\|\cdot\|_{E(\tau)}\right)$ into $\left(S(\tau), \mathcal{T}_{m}\right)$ is continuous and $S_{h}(\tau)^{+}$is closed in $S_{h}(\tau)$ (see Proposition 5.3), it is clear that 
$E_{h}(\tau)^{+}$is closed in $E_{h}(\tau)$. Therefore, $\left(E_{h}(\tau),\|\cdot\|_{E(\tau)}\right)$ is an ordered Banach space (for an exposition of the theory of ordered Banach spaces we refer the reader to e.g. [2]; see also Chapter $\mathrm{V}$ in the book [33]). The positive cone $E_{h}(\tau)^{+}$is generating (indeed, each $a \in E_{h}(\tau)$ can be decomposed as $a=a^{+}-$ $a^{-}$, where $a^{+}$and $a^{-}$belong to $E_{h}(\tau)^{+}$with $\left.\left\|a^{+}\right\|_{E(\tau)},\left\|a^{-}\right\|_{E(\tau)} \leq\|a\|_{E(\tau)}\right)$. Furthermore, the norm in $E_{h}(\tau)$ is monotone, that is, $0 \leq a \leq b$ in $E_{h}(\tau)$ implies that $\|a\|_{E(\tau)} \leq\|b\|_{E(\tau)}$. This implies in particular that $E_{h}(\tau)^{+}$is a normal cone. Consequently, any $\varphi$ in the (real) Banach space dual $E_{h}(\tau)^{*}$ can be decomposed as $\varphi=\varphi_{1}-\varphi_{2}$, with $\varphi_{1}, \varphi_{2} \geq 0$. In other words, the dual cone of $E_{h}(\tau)^{+}$is generating in $E_{h}(\tau)^{*}$. Moreover, a standard argument shows that any positive linear functional on $E_{h}(\tau)$ is automatically bounded.

Note that $E(\tau)$ is the complexification of $E_{h}(\tau)$, that is, $E(\tau)=E_{h}(\tau) \oplus$ $i E_{h}(\tau)$. Indeed, any $x \in E(\tau)$ can be written as $x=\operatorname{Re} x+i \operatorname{Im} x$, with $\operatorname{Re} x, \operatorname{Im} x \in E_{h}(\tau)$ (and $\left.\|\operatorname{Re} x\|_{E(\tau)},\|\operatorname{Im} x\|_{E(\tau)} \leq\|x\|_{E(\tau)}\right)$. This implies that $E_{h}(\tau)^{*}$ may be identified with a closed real subspace of $E(\tau)^{*}$. Indeed, let us call a functional $\varphi \in E(\tau)^{*}$ self-adjoint (or, hermitian) whenever $\varphi\left(x^{*}\right)=\overline{\varphi(x)}$ for all $x \in E(\tau)$ and denote by $E(\tau)_{h}^{*}$ the closed real subspace of $E(\tau)^{*}$ consisting of all self-adjoint functionals. It is easy to verify that the map $\left.\varphi \longmapsto \varphi\right|_{E_{h}(\tau)}$ defines an isometric isomorphism form $E(\tau)_{h}^{*}$ onto $E_{h}(\tau)^{*}$. Via this isomorphism we may identify $E(\tau)_{h}^{*}$ with $E_{h}(\tau)^{*}$. Furthermore, with this identification, we have $E(\tau)^{*}=E_{h}(\tau)^{*} \oplus i E_{h}(\tau)^{*}$. Indeed, any $\varphi \in E(\tau)^{*}$ can be written as $\varphi=\varphi_{1}+i \varphi_{2}$, where $\varphi_{1}, \varphi_{2} \in E_{h}(\tau)^{*}$ are given by

$$
\varphi_{1}(x)=\frac{1}{2}\left(\varphi(x)+\overline{\varphi\left(x^{*}\right)}\right), \varphi_{1}(x)=\frac{1}{2 i}\left(\varphi(x)-\overline{\varphi\left(x^{*}\right)}\right), x \in E(\tau)^{*} .
$$

This implies in particular that every $\varphi \in E(\tau)^{*}$ is a linear combination of four positive linear functionals.

As specific examples we mention the non-commutative $L_{p}$-spaces associated with $(\mathcal{M}, \tau)$, that is, the spaces $L_{p}(\tau)$ corresponding to $L_{p}(0, \infty)$, for $1 \leq$ $p \leq \infty$. The norm in $L_{p}(\tau)$ is usually denoted simply by $\|\cdot\|_{p}$. In particular, $L_{\infty}(\tau)=\mathcal{M}$ and $\|x\|_{\infty}=\|x\|_{B(\mathcal{H})}$ for all $x \in L_{\infty}(\tau)$ (see the remarks preceding Theorem 6.1).

If we take $\mathcal{M}=B(\mathcal{H})$ with standard trace, then the spaces $E(\tau)$ correspond to the so-called symmetrically normed ideals of compact operators, the theory of which is developed in detail in the book [18]. In particular, in this case $L_{p}(\tau)=\mathfrak{S}_{p}$ for $1 \leq p<\infty$, the $p$-Schatten ideals of compact operators.

It follows from (3) that any non-commutative Banach function space satisfies

$$
\left(L_{1} \cap L_{\infty}\right)(\tau) \subseteq E(\tau) \subseteq\left(L_{1}+L_{\infty}\right)(\tau)
$$

with continuous embeddings. It is clear that $\left(L_{1} \cap L_{\infty}\right)(\tau)=L_{1}(\tau) \cap L_{\infty}(\tau)$ and it can be shown that $\left(L_{1}+L_{\infty}\right)(\tau)=L_{1}(\tau)+L_{\infty}(\tau)$. The restriction of the trace $\tau$ to $\left(L_{1} \cap L_{\infty}\right)_{h}^{+}(\tau)$ is a positive linear functional and can be extended to a linear functional $\dot{\tau}$ on $\left(L_{1} \cap L_{\infty}\right)(\tau)$, satisfying $\dot{\tau}(|x|)=\|x\|_{1}$ for all $x \in$ $\left(L_{1} \cap L_{\infty}\right)(\tau)$. Using that $\left(L_{1} \cap L_{\infty}\right)(0, \infty)$ is dense in $L_{1}(0, \infty)$, it follows that $\left(L_{1} \cap L_{\infty}\right)(\tau)$ is dense in $L_{1}(\tau)$ (see e.g. Proposition 2.8 in [12]) and hence, $\dot{\tau}$ extends uniquely to a linear functional $\dot{\tau}: L_{1}(\tau) \rightarrow \mathbb{C}$. Moreover, $\dot{\tau}(|x|)=\|x\|_{1}$ for all $x \in L_{1}(\tau)$ and $\dot{\tau}$ is a positive functional on $L_{1}^{+}(\tau)$. For the details of this construction and further properties of this extended trace, which will be denoted again by $\tau$, we refer the reader to Section 3 of [12]. 
Next we discuss some aspects of the duality theory of these non-commutative spaces. As above, we assume that $E(0, \infty)$ is a symmetric Banach function space on $(0, \infty)$.

Definition 7.2 The Köthe dual space $E(\tau)^{\times}$of $E(\tau)$ is defined by

$$
E(\tau)^{\times}=\left\{y \in S(\tau): x y \in L^{1}(\tau) \quad \forall x \in E(\tau)\right\} .
$$

It is clear that $E(\tau)^{\times}$is a linear subspace of $S(\tau)$. It is not difficult to verify that $y \in E(\tau)^{\times}$if and only if $y x \in L^{1}(\tau)$ for all $x \in E(\tau)$. Moreover, if $y \in E(\tau)^{\times}$and $x \in E(\tau)$, then $\tau(x y)=\tau(y x)$. If, in addition, $x \geq 0$ and $y \geq 0$, then $\tau(x y) \geq 0$ (all these statements, and much more, can be found in Proposition 5.2 of $[12])$. For $y \in E(\tau)^{\times}$, we define the linear functional

$$
\varphi_{y}: E(\tau) \rightarrow \mathbb{C}, \varphi_{y}(x)=\tau(x y), x \in E(\tau)
$$

If $y \in E(\tau)^{\times}$and $y \geq 0$, then $\varphi_{y}$ is a positive functional, that is, $\varphi_{y}(x) \geq 0$ for all $x \in E_{h}^{+}(\tau)$. This observation can be used to show that the functional $\varphi_{y}$ is bounded for every $y \in E(\tau)^{\times}$. The map $\Phi: E(\tau)^{\times} \rightarrow E(\tau)^{*}$ is linear and injective (which follows from $\left.\left(L_{1} \cap L_{\infty}\right)(\tau) \subseteq E(\tau)\right)$. Now we define a norm $\|\cdot\|_{E(\tau)^{\times}}$on $E(\tau)^{\times}$by

$$
\|y\|_{E(\tau)^{\times}}=\left\|\varphi_{y}\right\|_{E(\tau)^{*}}, \quad y \in E(\tau)^{\times} .
$$

We say that $E(\tau)^{\times}$may be identified with a subspace of $E(\tau)^{*}$ via trace duality (which is given by (11). In the analysis of Köthe dual $E(\tau)^{\times}$the following result plays a crucial role (see [11], Proposition 5.3).

Proposition 7.3 If $y \in S(\tau)$, then $y \in E(\tau)^{\times}$if and only if

$$
\sup \left\{\int_{0}^{\infty} \mu(x ; t) \mu(y ; t) d t: x \in E(\tau),\|x\|_{E(\tau)} \leq 1\right\}<\infty
$$

and in this case we have

$$
\|y\|_{E(\tau)^{\times}}=\sup \left\{\int_{0}^{\infty} \mu(x ; t) \mu(y ; t) d t: x \in E(\tau),\|x\|_{E(\tau)} \leq 1\right\} .
$$

Using these observations, it can be shown that the normed linear space $\left(E(\tau)^{\times},\|\cdot\|_{E(\tau)^{\times}}\right)$has the following properties (see [12], Proposition 5.4):

(a). $\left(L_{1} \cap L_{\infty}\right)(\tau) \subseteq E(\tau)^{\times} \subseteq\left(L_{1}+L_{\infty}\right)(\tau)$, with continuous embeddings;

(b). the embedding of $\left(E(\tau)^{\times},\|\cdot\|_{E(\tau)^{\times}}\right)$into $\left(S(\tau), \mathcal{T}_{m}\right)$ is continuous;

(c). if $x \in S(\tau), y \in E(\tau)^{\times}$and $x \prec y$, then $x \in E(\tau)^{\times}$and $\|x\|_{E(\tau)^{\times}} \leq$ $\|y\|_{E(\tau)^{\times}}$

(d). if $\left\{y_{\alpha}\right\}$ is a net of positive elements in $E(\tau)^{\times}$such that $0 \leq y_{\alpha} \uparrow$ and $\sup _{\alpha}\left\|y_{\alpha}\right\|_{E(\tau)^{\times}}<\infty$, then there exists a positive element $y \in E(\tau)^{\times}$such that $y_{\alpha} \uparrow y$ and $\left\|y_{\alpha}\right\|_{E(\tau)} \times \uparrow\|y\|_{E(\tau)} \times$; 
(e). $E(\tau)^{\times}$is complete with respect to $\|\cdot\|_{E(\tau)^{\times}}$.

The important result for the identification of the Köthe dual $E(\tau)^{\times}$is the following theorem (see [12], Theorem 5.6).

Theorem 7.4 If $E=E(0, \infty)$ is a symmetric Banach function space on $(0, \infty)$ with Köthe dual space $E^{\times}$, then $E(\tau)^{\times}=E^{\times}(\tau)$ (with equality of norms).

The linear functionals $\varphi \in E(\tau)^{*}$ which correspond to elements $y \in E(\tau)^{\times}$ via trace duality (11) have a characterization which is analogous to the commutative case (see Theorem 2.10).

Theorem 7.5 ([12], Theorem 5.11) Suppose that $E=E(0, \infty)$ is a symmetric Banach function space on $(0, \infty)$. For $\varphi \in E(\tau)^{*}$ the following conditions are equivalent:

(i). $\varphi$ is normal, that is, $x_{\alpha} \downarrow 0$ in $E_{h}(\tau)$ implies that $\varphi\left(x_{\alpha}\right) \rightarrow 0$;

(ii). $\varphi$ is completely additive, that is, $p_{\alpha} \downarrow 0$ in $P(\mathcal{M})$ implies that $\varphi\left(x e_{\alpha}\right) \rightarrow 0$ and $\varphi\left(e_{\alpha} x\right) \rightarrow 0$ for all $x \in E(\tau)$;

(iii). there exists $y \in E(\tau)^{\times}$such that $\varphi(x)=\tau(x y)$ for all $x \in E(\tau)$ (that is, $\varphi=\varphi_{y}$ in the notation of (11)).

Via the same argument as used in the case of Banach lattices, it is easily see that every $\varphi \in E(\tau)^{*}$ is normal (briefly, $\left.E(\tau)^{*}=E(\tau)^{\times}\right)$if and only if the norm in $E(\tau)$ is order continuous, that is, $x_{\alpha} \downarrow 0$ in $E_{h}(\tau)$ implies that $\left\|x_{\alpha}\right\|_{E(\tau)} \downarrow 0$. Another relevant observation in this connection is the following (see [12], Proposition 3.6, and [7]).

Proposition 7.6 If the symmetric Banach function space $E=E(0, \infty)$ has order continuous norm, then the norm in $E(\tau)$ is also order continuous.

Proof. The order continuity of the norm in $E(0, \infty)$ implies that $\mu(f ; t) \rightarrow$ 0 as $t \rightarrow \infty$ and so, $E(\tau) \subseteq S_{0}(\tau)$ (see (10)). Consequently, if $x_{0} \geq x_{\alpha} \downarrow 0$ in $E_{h}(\tau)$, then it follows from Corollary 6.4 that $\mu\left(x_{\alpha} ; t\right) \downarrow 0$ for all $t>0$ and hence, $\left\|x_{\alpha}\right\|_{E(\tau)}=\left\|\mu\left(x_{\alpha}\right)\right\|_{E(0, \infty)} \downarrow 0$.

We illustrate the above results with some explicit examples. If we take for example $E=L_{p}(0, \infty)$, with $1 \leq p<\infty$, the $E$ has order continuous norm and so,

$$
L_{p}(\tau)^{*}=L_{p}(\tau)^{\times}=L_{p}^{\times}(\tau)=L_{q}(\tau)
$$

(identification via trace duality), where $p^{-1}+q^{-1}=1$. Similarly, $\mathcal{M}^{\times}=$ $L_{\infty}(\tau)^{\times}=L_{1}(\tau)$. Other examples are

$$
\begin{aligned}
& \left(L_{1}(\tau)+L_{\infty}(\tau)\right)^{\times}=\left(L_{1}+L_{\infty}\right)^{\times}(\tau)=\left(L_{1} \cap L_{\infty}\right)(\tau)=L_{1}(\tau) \cap L_{\infty}(\tau), \\
& \left(L_{1}(\tau) \cap L_{\infty}(\tau)\right)^{\times}=\left(L_{1} \cap L_{\infty}\right)^{\times}(\tau)=\left(L_{1}+L_{\infty}\right)(\tau)=L_{1}(\tau)+L_{\infty}(\tau) .
\end{aligned}
$$

Of course, similar examples may be given using Orlicz spaces, Lorentz and Marcinkiewicz spaces.

We end this section with an interesting decomposition theorem for functionals in the Banach space dual $E(\tau)^{*}$, as was obtained in [14]. Let us first 
consider the situation for a Banach function space $E$ on a (Maharam) measure space $(X, \Sigma, \nu)$. As before, we denote by $E_{n}^{*}$ the collection of all normal (that is, order continuous) linear functionals on $E$, which is a band in the Banach space dual $E^{*}$ (and may be identified with the Köthe dual $E^{\times}$). The disjoint complement of $E_{n}^{*}$ in $E^{*}$ will be denoted by $E_{s}^{*}$ (sometimes this band is also denoted by $E_{s n}^{*}$ ) and the elements of $E_{s}^{*}$ are termed singular (normal) linear functionals. Since $E^{*}=E_{n}^{*} \oplus E_{s}^{*}$, every $\varphi \in E^{*}$ has a unique decomposition $\varphi=\varphi_{n}+\varphi_{s}$, where $\varphi_{n} \in E_{n}^{*}$ and $\varphi_{s} \in E_{s}^{*}$ (and so, $\varphi_{n} \perp \varphi_{s}$ ). This decomposition can be viewed as an analogue of the so-called Yosida-Hewitt decomposition of measures). For the details we refer the reader to e.g. Chapter 12 in [40] or Chapter 1 in [1]. From the definition it is clear that a functional $\varphi \in E^{*}$ is singular if and only if it follows from $|\psi| \leq|\varphi|$ and $\psi \in E_{n}^{*}$ that $\psi=0$. Another useful characterization of singular functionals is that they vanish on large (order) ideals in $E$. To be more precise, an ideal (that is, absolutely solid linear subspace) $A \subseteq E$ is called order dense in $E$ if for every $0<u \in E$ there exists $v \in A$ such that $0<v \leq u$. With this terminology, a linear functional $\varphi \in E^{*}$ is singular if and only $\varphi=0$ on some order dense ideal in $E$ (see [26], Theorem 50.4; this result also follows from Theorem 90.5 in [40]). We note that for this characterization it is essential that $E_{n}^{*}$ separates the points of the Banach function space $E$ (for example, on the Banach lattice $C[0,1]$ the functional of integration is singular and strictly positive).

Now we consider such a decomposition for functionals on a space $E(\tau)$, where $(\mathcal{M}, \tau)$ is a semi-finite von Neumann algebra and $E=E(0, \infty)$ is a symmetric Banach function space on $(0, \infty)$. The concept of normal functional was already introduced in Theorem 7.5. A linear subspace $A \subseteq E(\tau)$ is called an order ideal if $A$ is generated by its positive elements and if it follows from $0 \leq b \leq a, a \in A$ and $b \in E_{h}(\tau)$ that $b \in A$. Such an ideal $A$ is called order dense in $\bar{E}(\tau)$ if for every $0<b \in E_{h}(\tau)$ there exists $a \in A$ such that $0<a \leq b$. A linear functional $\varphi \in E(\tau)^{*}$ is said to be singular whenever $\varphi$ vanishes on some order dense ideal in $E(\tau)$. Evidently, this notion of singularity agrees with the one for Banach function spaces, as follows from the above discussion. If $E=L_{\infty}(0, \infty)$, and so $E(\tau)=\mathcal{M}$, it also agrees with the usual definition of a singular functional on a von Neumann algebra (see e.g. [34], Section III.2 or [22], Section 10.1), as follows from [14], Proposition 2.1, in combination with [34], Theorem III.3.8. Now we are in a position to formulate the decomposition theorem for elements of $E(\tau)^{*}$.

Theorem 7.7 ([14], Corollary 2.5) If $(\mathcal{M}, \tau)$ is a semi-finite von Neumann algebra and $E=E(0, \infty)$ is a fully symmetric Banach function space on $(0, \infty)$, then every $\varphi \in E(\tau)^{*}$ has a unique decomposition $\varphi=\varphi_{n}+\varphi_{s}$, where $\varphi_{n}$ is normal and $\varphi_{s}$ is singular.

For further details and interesting applications of this result, we refer the reader to [14].

\section{Operator functions}

As we have seen in the previous section, there are many results concerning non-commutative Banach function spaces which are analogous to the commutative theory (although most of the proofs are quite different!). However, there 
are some aspects of the non-commutative theory which are essentially different from the commutative situation. We shall illustrate this with some results concerning so-called operator functions. By an operator function we mean a map $a \longmapsto f(a)$, where $f: \mathbb{R} \rightarrow \mathbb{R}$ is an appropriate Borel function and the (noncommutative) variable $a$ belongs to $E_{h}(\tau)$. If $f: \mathbb{R} \rightarrow \mathbb{R}$ is continuous, then we know by Theorem 5.12 that the map $a \longmapsto f(a)$ from $S_{h}(\tau)$ into itself, is continuous with respect to the measure topology. But, here we will be interested in Lipschitz-type norm estimates. To be more precise, we consider the following problem: under which assumptions, on the Banach function space $E=E(0, \infty)$ and on the function $f$, does there exists a constant $C>0$ (depending on $E$ and $f)$ such that $\|f(a)-f(b)\|_{E(\tau)} \leq C\|a-b\|_{E(\tau)}$ for all $a, b \in E_{h}(\tau)$ ?

Let us say first a few words about the commutative situation. Suppose that $E$ is any Banach function space on a (Maharam) measure space $(X, \Sigma, \nu)$ and let $a \in E$ be real valued (we use here the symbol $a$ for a function to keep the analogy with the above discussion). We may represent $a$ by its spectral integral $a=\int_{\mathbb{R}} \lambda d e^{a}(\lambda)$ as in (4). Note that we may consider $a$ as a self-adjoint operator on the Hilbert space $L_{2}(\nu)$, acting via multiplication. The spectral measure of $a$ is then given by $e^{a}(B)=\chi_{a^{-1}(B)}$ for all Borel sets $B \subseteq \mathbb{R}$. If $f: \mathbb{R} \rightarrow \mathbb{R}$ is a Borel function, then $f(a)$ is defined by

$$
f(a)=\int_{\mathbb{R}} f(\lambda) d e^{a}(\lambda)
$$

(see (5)). Approximating $f$ by simple functions, it is not difficult to see that $f(a)=f \circ a$, the composition of $f$ and $a$ ). Now suppose that the function $f$ is Lipschitz continuous, that is, there exists a constant $C>0$ such that $|f(\lambda)-f(\mu)| \leq C|\lambda-\mu|$ for all $\lambda, \mu \in \mathbb{R}$. If $a, b \in E$ are real valued, then

$$
|f(a)(x)-f(b)(x)|=|f(a(x))-f(b(x))| \leq C|a(x)-b(x)|, \quad x \in X,
$$

and hence,

$$
|f(a)-f(b)| \leq C|a-b| .
$$

Since $E$ is an ideal in $L_{0}(\nu)$ and the norm on $E$ is absolutely monotone, it follows that $f(a)-f(b) \in E$ and $\|f(a)-f(b)\|_{E} \leq C\|a-b\|_{E}$. This argument shows that in the commutative situation, it is more or less evident that Lipschitz continuity of $f$ implies that the corresponding "operator function" is also Lipschitz continuous (with the same constant, independent of $E$ ). The crucial estimate is of course inequality (12). In the non-commutative situation, inequalities like (12) are not valid in general (if $a$ and $b$ do not commute) and, as it turns out, a Lipschitz continuity of $f$ is in general not enough to guarantee that the corresponding operator function satisfies a Lipschitz estimate.

As a special case, let us first consider the absolute value mapping corresponding to the function $f(\lambda)=|\lambda|$. In [13] the following result has been obtained.

Theorem 8.1 Suppose that $1<p<\infty$ and let $(\mathcal{M}, \tau)$ be a semi-finite von Neumann algebra. If $x, y \in S(\tau)$ such that $x-y \in L_{p}(\tau)$, then $|x|-|y| \in L_{p}(\tau)$ and

$$
\||x|-|y|\|_{p} \leq C_{p}\|x-y\|_{p},
$$

where $C_{p}>0$ is a constant only depending on $p$. 
In the case $\mathcal{M}=B(\mathcal{H})$, with standard trace (and so, $L_{p}(\tau)=\mathfrak{S}_{p}$, the $p$-Schatten ideal), the above result was obtained by E.B. Davies in [8] (see also [5]). Moreover, it was shown in [8] that an estimate like (13) fails for $p=1, \infty$. We like to point out that it is sufficient to prove the above theorem for selfadjoint elements $x$ and $y$ only. Indeed, the general case is then obtained from this special case by considering the von Neumann algebra $M_{2}(\mathbb{C}) \otimes \mathcal{M}$, of all $2 \times 2$-matrices with entries in $\mathcal{M}$, and applying the result to the self-adjoint operators

$$
\left[\begin{array}{cc}
0 & x^{*} \\
x & 0
\end{array}\right],\left[\begin{array}{ll}
0 & y^{*} \\
y & 0
\end{array}\right] .
$$

We leave the verification to the reader.

Furthermore, the result of Theorem 8.1 can be extended via interpolation techniques to a much larger class of spaces than the $L_{p}$-spaces. In fact, in [13], Theorem 3.4, it was shown that, if $E=E(0, \infty)$ is a symmetric Banach function space which is an $\left(L_{p}, L_{q}\right)$-interpolation space for some $1<p \leq q<\infty$, then there exists a constant $C_{E}>0$ (only depending on the space $E$ ) such that $\||x|-|y|\|_{E(\tau)} \leq\|x-y\|_{E(\tau)}$ for all $x, y \in E(\tau)$ with $x-y \in E(\tau)$, for all semi-finite von Neumann algebras $(\mathcal{M}, \tau)$ (and actually, this property characterizes the Banach function spaces which are $\left(L_{p}, L_{q}\right)$-interpolation space for some $1<p \leq q<\infty)$.

Finally we say a few words about more general operator functions $a \longmapsto$ $f(a), a \in E_{h}(\tau)$. For sake of simplicity we shall not state the results in full generality, but single out some important special cases (which follows from [29], Corollary 7.5 in combination with Proposition 8.5).

Theorem 8.2 Suppose that $1<p<\infty$, let $(\mathcal{M}, \tau)$ be a semi-finite von Neumann algebra and $f: \mathbb{R} \rightarrow \mathbb{R}$ be a function with weak derivative $f^{\prime}$ which is of bounded variation. There exists a constant $C_{p, f}>0$ (only depending on $p$ and the function $f$ ), such that

$$
\|f(a)-f(b)\|_{p} \leq C_{p, f}\|a-b\|_{p}
$$

for all $a, b \in S_{h}(\tau)$ with $a-b \in L_{p}(\tau)$.

The function $f(\lambda)=|\lambda|$ satisfies the conditions of the above theorem and so, the result of Theorem 8.1 may be obtained via Theorem 8.2. Furthermore, also Theorem 8.2 actually holds for $\left(L_{p}, L_{q}\right)$-interpolation spaces with $1<p, q<\infty$. In the paper [30] several results concerning the (Gâteaux) differentiability of operator functions have been obtained. All these results depend on the theory of so-called double operator integrals, originated by Birman and Solomyak in the setting of trace ideals and extended in [29] to the general setting of semi-finite von Neumann algebras. These double operator integrals and their relation to the $U M D$-property and $R$-bounded collections of operators, have been discussed also in detail in [38].

\section{References}

[1] C.D. Aliprantis, O. Burkinshaw, Positive Operators, Academic Press, Orlando, 1985. 
[2] C.J.K. Batty, D.W. Robinson, Positive one-parameter semigroups on ordered Banach spaces, Acta Appl. Math. 1 (1984), 221-296.

[3] C. Bennett, R. Sharpley, Interpolation of Operators, Academic Press, Orlando, 1988.

[4] M.S̆. Birman, M.Z. Solomyak, Spectral theory of selfadjoint operators in Hilbert space, D. Reidel Publishing Co., Dordrecht, 1987.

[5] M.S̆. Birman, M.Z. Solomyak, Operator integration, perturbations and commutators, Zap. Nauchn. Sem. Leningrad. Otdel. Mat. Inst. Steklov. (LOMI), Issled. Linein. Teorii Funktsii. 17 (1989), 34-66.

[6] A.P. Calderón, Spaces between $L^{1}$ and $L^{\infty}$ and the theorem of Marcinkiewicz, Studia Math. 26 (1966), 273-299.

[7] V.I. Chilin, F.A. Sukochev, Symmetric spaces on semi-finite von Neumann algebras, Dokl. Akad. Nauk. SSSR 13 (1990), 811-815 (Russian).

[8] E.B. Davies, Lipschitz continuity of functions of operators in the Schatten classes, J. London Math. Soc. 37 (1988), 148-157.

[9] J. Dixmier, Von Neumann Algebras, North-Holland Mathematical Library, Vol. 27, North-Holland, Amsterdam, 1981.

[10] P.G. Dodds, T.K. Dodds, B. de Pagter, Non-commutative Banach function spaces, Math. Z. 201 (1989), 583-597.

[11] P.G. Dodds, T.K. Dodds, B. de Pagter, A general Marcus inequality, Proc. Centre Math. Anal. Austral. Nat. Univ. 24 (1989), 47-57.

[12] Peter G. Dodds, Theresa K.-Y. Dodds, Ben de Pagter, Non-commutative Köthe duality, Trans. Amer. Math. Soc. 339 (1993), 717-750.

[13] P.G. Dodds, T.K. Dodds, B. de Pagter, F.A. Sukochev, Lipschitz Continuity of the Absolute Value and Riesz Projections in Symmetric Operator Spaces, J. of Functional Analysis 148 (1997), 28-69.

[14] P.G. Dodds, T.K. Dodds, F.A. Sukochev, O.Ye. Tikhonov, A Noncommutative Yosida-Hewitt Theorem and Convex Sets of Measurable Operators Closed Locally in Measure, Positivity 9 (2005), 457-484.

[15] Th. Fack, H. Kosaki, Generalized s-numbers of $\tau$-measurable operators, Pacific J. Math. 123 (1986), 269-300.

[16] K. Fan, Maximum properties and inequalities for the eigenvalues of completely continuous operators, Proc. Nat. Acad. Sci. U.S.A. 37 (1951),760766 .

[17] D. H. Fremlin, Measure Theory, Volume 3: Measure Algebras, Torres Fremlin, Colchester, 2002.

[18] I.C. Gohberg, M.G. Krein, Introduction to the Theory of Linear Nonselfadjoint Operators, Translations of Mathematical Monographs, Vol. 18, AMS, Providence, R.I., 1969. 
[19] A. Grothendieck, Réarrangements de fonctions et inegalités de convexité dans les algèbres de von Neumann muni d'une trace, Seminaire Bourbaki, 1955, 113-01-113-13.

[20] P.R. Halmos, A Hilbert Space Problem Book, 2nd Ed., Graduate Texts in Math., Springer-Verlag, New York-Heidelberg-Berlin, 1982.

[21] R.V. Kadison, J.R. Ringrose, Fundamentals of the theory of operator algebras, Volume I: Elementary Theory, Academic Press, New York, 1983.

[22] R.V. Kadison, J.R. Ringrose, Fundamentals of the theory of operator algebras, Volume II: Advanced Theory, Academic Press, Orlando, 1986.

[23] T. Kato, Perturbation Theory for Linear Operators, Classics in Mathematics, Springer-Verlag, Berlin-Heidelberg-New York, 1995.

[24] S.G. Krein, Ju.I. Petunin, E.M. Semenov, Interpolation of Linear Operators, Translations of Math. Monographs, Vol. 54, Amer. Math. Soc., Providence, 1982.

[25] G.G. Lorentz and T. Shimogaki, Interpolation theorems for operators in function spaces, J. Functional An. 2 (1968), 31-51.

[26] W.A.J. Luxemburg, Notes on Banach function spaces XV , Indag. Math. 27 (1965), 415-446.

[27] W.A.J. Luxemburg, Rearrangement invariant Banach function spaces, Proc. Sympos. in Analysis, Queen's Papers in Pure and Appl. Math. 10(1967), 83-144.

[28] A.S. Markus, The eigen- and singular values of the sum and product of linear operators, Russian Math. Surveys 19 (1964) 91-120.

[29] B. de Pagter, F.A. Sukochev, H. Witvliet, Double Operator Integrals, J. of Functional Analysis 192 (2002), 52-111.

[30] B. de Pagter, F.A. Sukochev, Differentiation of operator functions in noncommutative $L_{p}$-spaces, J. of Functional Analysis 212 (2004), 28-75.

[31] B. de Pagter, F.A. Sukochev, Commutator estimates and $\mathbb{R}$-flows in..........TO APPEAR.

[32] F. Riesz, B. Sz.-Nagy, Functional Analysis, Frederick Ungar Publishing Co., New York, 1955.

[33] H.H. Schaefer (with M.P. Wolff), Topological Vector Spaces (2nd Edition), Springer-Verlag, New York, 1999.

[34] M. Takesaki, Theory of Operator Algebras I, Springer-Verlag, BerlinHeidelberg-New York, 1979.

[35] M. Takesaki, Theory of Operator Algebras II, Springer-Verlag, BerlinHeidelberg-New York, 2003.

[36] M. Terp, $L_{p}$-spaces associated with von Neumann algebras, Copenhagen University, 1981. 
[37] O.Ye. Tikhonov, Continuity of operator functions in topologies connected with a trace on a von Neumann algebra, Izv. Vyssh. Uchebn. Zaved. Mat. (1987), 77-79 (in Russian; translated in Sov. Math. (Iz. VUZ) 31 (1987), 110-114.

[38] H. Witvliet, Unconditional Schauder decompositions and multiplier theorems, Ph.D. thesis, Delft University of Technology, 2000.

[39] A.C. Zaanen, Integration, North-Holland Publishing Company, Amsterdam, 1967.

[40] A.C. Zaanen, Riesz Spaces II, North-Holland Publishing Company, Amsterdam-New York-Oxford, 1983.

Delft Institute of Applied Mathematics,

Faculty EEMCS,

Delft University of Technology,

P.0. Box 5031, 2600 GA Delft,

The Netherlands.

b.depagter@tudelft.nl 